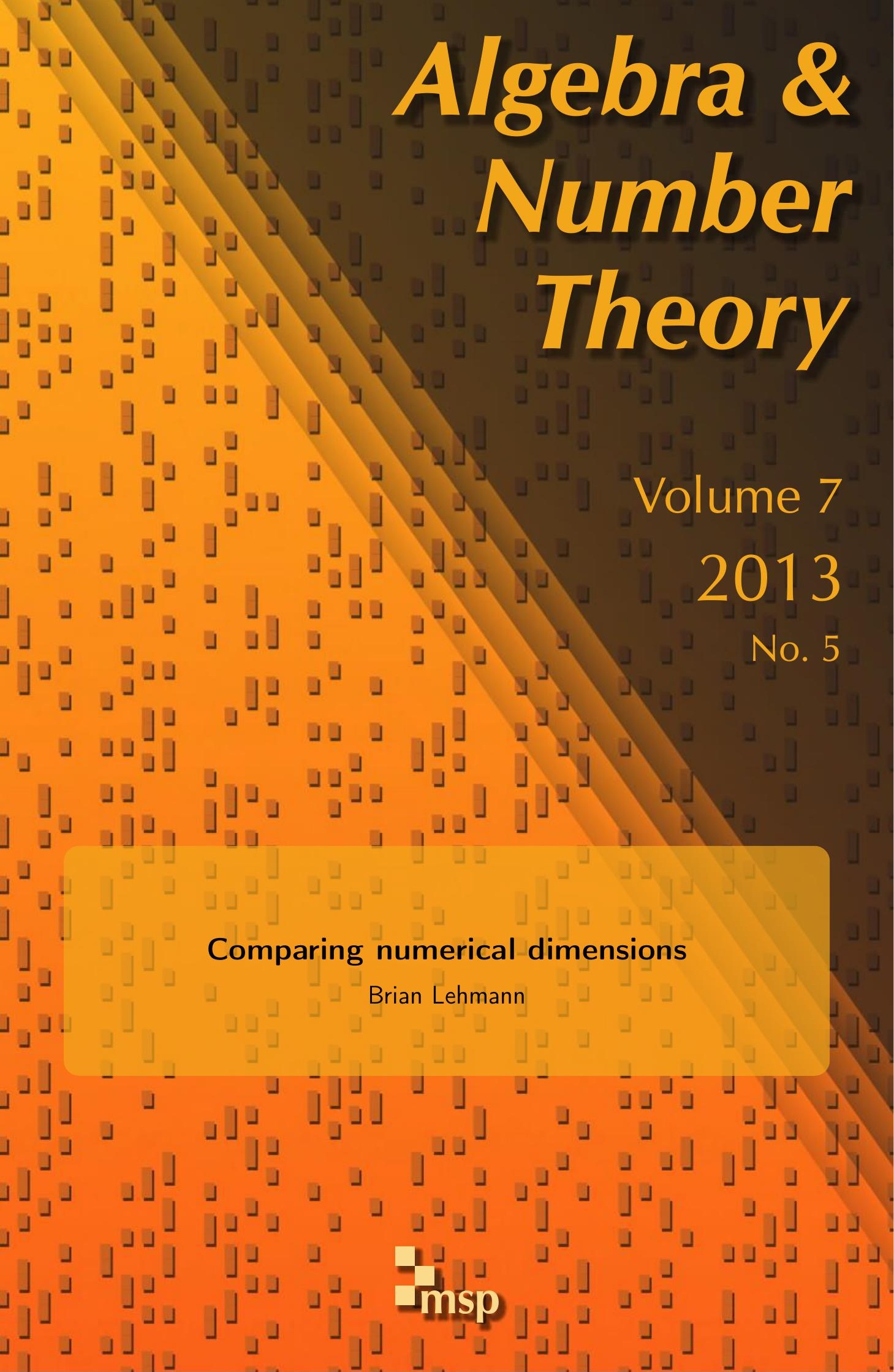




\title{
Comparing numerical dimensions
}

\author{
Brian Lehmann
}

The numerical dimension is a numerical measure of the positivity of a pseudoeffective divisor $L$. There are several proposed definitions of the numerical dimension due to Nakayama and Boucksom et al. We prove the equality of these notions and give several additional characterizations. We also prove some new properties of the numerical dimension.

\section{Introduction}

Suppose that $X$ is a smooth complex projective variety and $L$ is an effective divisor. An important principle in birational geometry is that the geometry of $L$ is captured by the asymptotic behavior of the spaces $H^{0}\left(X, O_{X}(m L)\right)$ as $m$ increases. When $L$ is a big divisor, this asymptotic behavior has close ties to the cohomological and numerical properties of $L$. These connections have been applied profitably in many situations in birational geometry, most notably in the minimal model program.

However, when $L$ is an effective divisor that is not big, these close relationships no longer hold. In order to understand the interplay between numerical and asymptotic properties, Kawamata [1985] defined the numerical dimension of a nef divisor. Nakayama [2004] and Boucksom et al. [2012] proposed several different extensions of this notion to pseudoeffective divisors. Our goal is to give a unifying framework for the numerical dimension by proving the equality of these definitions and giving other natural descriptions as well. We also describe some new properties of the numerical dimension. The crucial perspectives are the following:

(1) The numerical dimension measures the asymptotic behavior of $L$ when it is perturbed by adding a small ample divisor $\epsilon A$.

(2) The numerical dimension measures the largest dimension of a subvariety $W \subset X$ such that $L$ is positive along $W$. An important subtlety is that one should not simply consider $\left.L\right|_{W}$ but should "remove" contributions of the base locus of $L$.

This material is based upon work supported under a National Science Foundation Graduate Research Fellowship.

MSC2010: 14C20.

Keywords: divisor, numerical dimension. 
Since some of the definitions used in the main theorem are rather technical, we simply give references here. We will describe in Section 1A the intuition behind the theorem. The notation $\mathbf{B}_{-}(L)$ denotes the diminished base locus defined in Section $2 \mathrm{~A}, \operatorname{vol}_{X \mid W}$ denotes the restricted volume defined in Section 2D, $P_{\sigma}(-)$ denotes the divisorial Zariski decomposition defined in Section 3, and $\langle-\rangle$ denotes the restricted positive product defined in Section 4.

Theorem 1.1. Let $X$ be a normal projective variety over $\mathbb{C}$, and let $L$ be a pseudoeffective $\mathbb{R}$-Cartier $\mathbb{R}$-Weil divisor. In the following, A will denote some fixed sufficiently ample $\mathbb{Z}$-divisor, and $W$ will range over all subvarieties of $X$ not contained in $\mathbf{B}_{-}(L) \cup \operatorname{Supp}(L) \cup \operatorname{Sing}(X)$. The following quantities coincide:

Perturbed growth condition:

(1) $\max \left\{k \in \mathbb{Z}_{\geq 0} \mid \limsup _{m \rightarrow \infty} h^{0}\left(X, \mathscr{O}_{X}(\lfloor m L\rfloor+A)\right) / m^{k}>0\right\}$.

Volume conditions:

(2) $\max \left\{k \in \mathbb{Z}_{\geq 0} \mid \exists C>0\right.$ such that $C t^{n-k}<\operatorname{vol}(L+t A)$ for all $\left.t>0\right\}$.

(3) $\max \left\{\operatorname{dim} W \mid \lim _{\epsilon \rightarrow 0} \operatorname{vol}_{X \mid W}(L+\epsilon A)>0\right\}$.

(4) $\max \left\{\operatorname{dim} W \mid \inf _{\phi: Y \rightarrow X} \operatorname{vol}_{\widetilde{W}}\left(\left.P_{\sigma}\left(\phi^{*} L\right)\right|_{\widetilde{W}}\right)>0\right\}$, where $\phi$ varies over all birational maps such that no exceptional center contains $W$ and $\widetilde{W}$ denotes the strict transform of $W$.

Positive product conditions:

(5) $\max \left\{k \in \mathbb{Z}_{\geq 0} \mid\left\langle L^{k}\right\rangle \neq 0\right\}$.

(6) $\max \left\{\operatorname{dim} W \mid\left\langle L^{\operatorname{dim} W}\right\rangle_{X \mid W}>0\right\}$.

Seshadri-type condition:

(7) $\min \left\{\operatorname{dim} W \mid \phi^{*} L-\epsilon E\right.$ is not pseudoeffective for any $\left.\epsilon>0\right\}$, where $\phi$ denotes the blow-up $\phi: B l_{W} X \rightarrow X$ and $E$ denotes the Cartier divisor on $B l_{W} X$ such that $\mathscr{O}_{B l_{W} X}(-E)=\phi^{-1} \mathscr{I}_{W} \cdot \mathscr{O}_{B l_{W} X}$. (By convention, if $L$ is big, we interpret this expression as returning $\operatorname{dim} X$.)

This common quantity is known as the numerical dimension of $L$ and is denoted $v(L)$. It only depends on the numerical class of $L$.

The definitions $\kappa_{\sigma}$ and $\kappa_{v}$ of [Nakayama 2004, pp. 174 and 181] are listed as (1) and (7), respectively; the definition $v$ of [Boucksom et al. 2012] is listed as (5). When $L$ is numerically effective, this definition agrees with the definition of [Kawamata 1985].

Remark 1.2. The numerical dimension also admits a natural interpretation with respect to separation of jets, reduced volumes, and the other invariants considered in [Ein et al. 2009]. 
The numerical dimension is natural from the viewpoint of birational geometry. It is established in [Nakayama 2004] that for a pseudoeffective divisor $L$,

- $0 \leq v(L) \leq \operatorname{dim} X$,

- $v(L)=\operatorname{dim} X$ if and only if $L$ is big and $v(L)=0$ if and only if $P_{\sigma}(L) \equiv 0$,

- $\kappa(L) \leq v(L)$, and

- if $\phi: Y \rightarrow X$ is a surjective morphism, then $v\left(\phi^{*} L\right)=v(L)$.

We prove two additional basic properties, answering a question of Nakayama:

- We have $v(L)=v\left(P_{\sigma}(L)\right)$.

- Fix some sufficiently ample $\mathbb{Z}$-divisor $A$. Then there are positive constants $C_{1}$ and $C_{2}$ such that

$$
C_{1} m^{\nu(L)}<h^{0}\left(X, O_{X}(\lfloor m L\rfloor+A)\right)<C_{2} m^{\nu(L)}
$$

for every sufficiently large $m$.

The properties of $v(L)$ will be discussed in more depth in Section 6.

1A. Intuitive description. We now turn to an intuitive description of several of the definitions in Theorem 1.1. Classically, one measures the positivity of a divisor using the rate of growth of sections of $H^{0}\left(X, O_{X}(m L)\right)$ as $m$ increases. More precisely, the Iitaka dimension is defined as

$$
\kappa(L)=\max \left\{k \in \mathbb{Z}_{\geq 0} \mid \limsup _{m \rightarrow \infty} \frac{h^{0}\left(X, \mathscr{O}_{X}(\lfloor m L\rfloor)\right)}{m^{k}}>0\right\} .
$$

(If $H^{0}\left(X, \mathcal{O}_{X}(\lfloor m L\rfloor)\right)=0$ for every $m$, we set $\kappa(L)=-\infty$.) To obtain a numerical invariant, we must instead consider sections of $m L+A$ for some sufficiently ample divisor $A$. Thus, definition (1) indicates that $v(L)$ can be viewed as a numerical analogue of the Iitaka dimension.

Another way to calculate the positivity of $L$ is to use intersection products. [Kawamata 1985] defined the numerical dimension of a numerically effective divisor $L$ as

$$
v(L):=\max \left\{k \in \mathbb{Z}_{\geq 0} \mid L^{k} \cdot A^{n-k} \neq 0\right\}
$$

for some (thus any) ample divisor $A$. The naïve extension of this definition to pseudoeffective divisors does not work as the diminished base locus of $L$ might contribute positively to this intersection and distort the measurement. The positive product of [Boucksom et al. 2012] gives a precise method of taking intersection products while discounting these contributions. Definition (5) shows that $v(L)$ can be defined as in [Kawamata 1985] by replacing the intersection product by the positive product. 
A third way to measure the positivity of a divisor is the volume: if $n=\operatorname{dim} X$,

$$
\operatorname{vol}(L):=\limsup _{m \rightarrow \infty} \frac{h^{0}\left(X, \mathscr{O}_{X}(m L)\right)}{m^{n} / n !} .
$$

Conceptually, we can view the volume as a loose analogue of the top self-intersection of $L$. While this latter quantity does not usually yield geometric information, the volume is a useful alternative that still shares many of the desirable properties of intersection products. It is shown in [Lazarsfeld and Mustaţă 2009; Boucksom et al. 2009] that vol is a differentiable function on the space of big $\mathbb{R}$-Cartier divisors. Definition (2) demonstrates that $v(L)$ controls the derivative of vol near $L$.

1B. Restricted numerical dimension. It is useful to study not only numerical invariants on $X$ but also restricted versions that measure positivity along a subvariety $V$. We will define a restricted numerical dimension of $L$ along a subvariety $V$ of $X$. Just as in the nonrestricted case, the restricted numerical dimension should measure the maximal dimension of a very general subvariety $W \subset V$ such that the "positive restriction" of $L$ is big along $W$.

Definition 1.3. Let $X$ be a smooth variety, $V$ a subvariety, and $L$ a pseudoeffective $\mathbb{R}$-divisor such that $V \not \subset \mathbf{B}_{-}(L)$. Fix an ample divisor $A$. We define the restricted numerical dimension $v_{X \mid V}(L)$ to be

$$
v_{X \mid V}(L):=\max \left\{\operatorname{dim} W \mid \lim _{\epsilon \rightarrow 0} \operatorname{vol}_{X \mid W}(L+\epsilon A)>0\right\},
$$

where $W$ ranges over smooth subvarieties of $V$ not contained in $\mathbf{B}_{-}(L)$. The restricted numerical dimension is an invariant of the numerical class of $L$.

The restricted numerical dimension satisfies (slightly weaker) analogues of Theorems 1.1 and 6.7. For numerically effective divisors, we obtain nothing new because $v_{L \mid V}(L)=v_{V}\left(\left.L\right|_{V}\right)$. Nevertheless, the restricted numerical dimension plays an important role in understanding the geometry of a pseudoeffective divisor $L$.

1C. Organization. The paper is organized as follows. Section 3 is devoted to the study of the divisorial Zariski decomposition, giving the technical background for the rest of the paper. Sections 4 and 5 prove some basic facts about the invariants of Theorem 1.1. We then turn to the proof of Theorem 1.1 in Section 6. Section 7 is devoted to a discussion of the restricted numerical dimension.

\section{Preliminaries}

All schemes will lie over the base field $\mathbb{C}$. A variety will always be an irreducible reduced projective scheme. The ambient variety $X$ is assumed to be normal unless otherwise noted. The term "divisor" will always refer to an $\mathbb{R}$-Cartier $\mathbb{R}$-Weil divisor. Let $N^{p}(X)$ denote the $\mathbb{R}$-vector space of codimension- $p$ cycles quotiented out by 
those numerically equivalent to 0 , and $C D(X)$ will denote the $\mathbb{R}$-vector space of Cartier divisors quotiented out by those that have degree 0 along every irreducible curve.

2A. Base loci. Let $L$ be a pseudoeffective divisor. The $\mathbb{R}$-stable base locus of $L$ is defined to be

$$
\mathbf{B}_{\mathbb{R}}(L):=\bigcap\left\{\operatorname{Supp}(D) \mid D \geq 0 \text { and } D \sim_{\mathbb{R}} L\right\} .
$$

When $L$ is not $\mathbb{R}$-linearly equivalent to an effective divisor, we use the convention that $\mathbf{B}_{\mathbb{R}}(L)=X$. The $\mathbb{R}$-stable base locus is always a Zariski-closed subset of $X$; we do not associate any scheme structure to it.

We obtain a much better behaved invariant by perturbing by an ample divisor. This approach to invariants was first considered in [Nakamaye 2000] and was studied systematically in [Ein et al. 2006].

Definition 2.1. Let $L$ be a pseudoeffective divisor. The augmented base locus of $L$ is

$$
\mathbf{B}_{+}(L):=\bigcap_{A \text { ample }} \mathbf{B}_{\mathbb{R}}(L-A) .
$$

Note that $\mathbf{B}_{+}(L) \supset \mathbf{B}_{\mathbb{R}}(L)$. [Ein et al. 2006, Corollary 1.6] verifies that the augmented base locus is equal to $\mathbf{B}_{\mathbb{R}}(L-A)$ for any sufficiently small ample divisor $A$. Thus, $\mathbf{B}_{+}(L)$ is a Zariski-closed subset of $X$, and it only depends on the numerical class of $L$.

For the second variant, we add on a small ample divisor.

Definition 2.2. Let $L$ be a pseudoeffective divisor. The diminished base locus of $L$ is

$$
\mathbf{B}_{-}(L):=\bigcup_{A \text { ample }} \mathbf{B}_{\mathbb{R}}(L+A) .
$$

Remark 2.3. Although Nakayama [2004] uses a different definition, it is equivalent to ours by his Theorem V.1.3.

Proposition 1.15 of [Ein et al. 2006] checks that the diminished base locus only depends on the numerical class of $L$. Unlike the augmented base locus, the diminished base locus is probably not a Zariski-closed subset (although no examples are known of such pathological behavior). However, it is a countable union of closed subsets by the following theorem:

Theorem 2.4 [Nakayama 2004, Theorem V.1.3]. Let X be a smooth variety, and let $L$ be a pseudoeffective divisor. There is an ample divisor A such that

$$
\mathbf{B}_{-}(L)=\bigcup_{m} \mathrm{Bs}(\lceil m L\rceil+A),
$$

where Bs denotes the (set-theoretic) base locus. 
From [Nakayama 2004] we know $\mathbf{B}_{-}(L)$ is invariant under surjective morphisms.

Proposition 2.5. Let $\phi: Y \rightarrow X$ be a surjective morphism from a normal variety $Y$ onto a normal variety $X$. Suppose that $L$ is a pseudoeffective divisor on $X$. Then we have an equality of sets

$$
\phi^{-1} \mathbf{B}_{-}(L) \cup \phi^{-1} \operatorname{Sing}(X)=\mathbf{B}_{-}\left(\phi^{*} L\right) \cup \phi^{-1} \operatorname{Sing}(X) .
$$

Proof. Fix an ample divisor $H$ on $Y$ and an ample divisor $A$ on $X$. We have

$$
\begin{array}{rlr}
\phi^{-1} \mathbf{B}_{-}(L) & =\phi^{-1}\left(\bigcup_{m} \mathbf{B}_{\mathbb{R}}\left(L+\frac{1}{m} A\right)\right) \quad \text { by [Ein et al. 2006, Remark 1.20] } \\
& =\bigcup_{m} \mathbf{B}_{\mathbb{R}}\left(\phi^{*}\left(L+\frac{1}{m} A\right)\right) \\
& \supset \bigcup_{m} \mathbf{B}_{\mathbb{R}}\left(\phi^{*}\left(L+\frac{1}{m} A\right)+\frac{1}{m} H\right) \\
& =\mathbf{B}_{-}\left(\phi^{*} L\right) \quad \text { by [Ein et al. 2006, Remark 1.20]. }
\end{array}
$$

This proves the inclusion $\supset$. Furthermore, the same argument shows that it suffices to prove the reverse inclusion $\subset$ after replacing $Y$ by any higher birational model.

We next reduce to the case where $X$ and $Y$ are smooth. Let $\psi: \widetilde{X} \rightarrow X$ denote a resolution that is an isomorphism away from $\operatorname{Sing}(X)$. Suppose that the closed point $\tilde{x} \notin \mathbf{B}_{-}\left(\phi^{*} L\right) \cup \phi^{-1} \operatorname{Sing}(X)$. Fix an ample divisor $\widetilde{A}$ on $\widetilde{X}$, and choose an ample divisor $A$ on $X$ so that $\phi^{*} A-\widetilde{A}$ is an effective divisor $E$. Since $\tilde{x}$ is not contained in the $\psi$-exceptional locus, we may also ensure that $\tilde{x} \notin \operatorname{Supp}(E)$. Then

$$
\tilde{x} \notin \mathbf{B}_{\mathbb{R}}\left(\phi^{*}(L)+\epsilon H+\epsilon E\right)=\phi^{-1} \mathbf{B}_{\mathbb{R}}\left(\phi^{*}(L+\epsilon A)\right)
$$

for any $\epsilon>0$, showing that

$$
\psi^{-1} \mathbf{B}_{-}(L) \cup \psi^{-1} \operatorname{Sing}(X)=\mathbf{B}_{-}\left(\psi^{*} L\right) \cup \psi^{-1} \operatorname{Sing}(X) .
$$

As discussed earlier, we may verify the desired equality of sets by replacing $Y$ by a smooth birational model that dominates $\tilde{X}$. Thus, we have reduced to the case when both $X$ and $Y$ are smooth.

[Nakayama 2004, Lemmas III.2.3 and III.5.15] together show that for a smooth variety $Z$ and a pseudoeffective divisor $M$ on $Z$, a closed point $z \in Z$ is contained in $\mathbf{B}_{-}(M)$ if and only if, for every birational map $\psi: W \rightarrow Z$ from a smooth variety $W$ and every $\psi$-exceptional divisor $E$ with $\psi(E)=z$, we have $E \subset \mathbf{B}_{-}\left(\psi^{*} L\right)$. This immediately implies the inclusion $\subset$ when both $X$ and $Y$ are smooth.

2B. V-pseudoeffective cone and V-big cone. The perturbed base loci can be used to describe when a divisor $L$ sits in "general position" with respect to a subvariety $V$. 
Definition 2.6. Suppose that $V \subset X$ is a subvariety. We define the $V$-pseudoeffective cone $\operatorname{Psef}_{V}(X)$ to be the cone in $C D(X)$ generated by classes of divisors $L$ with $V \not \subset \mathbf{B}_{-}(L)$. We define the $V$-big cone $\operatorname{Big}_{V}(X)$ to be the cone generated by classes of divisors $L$ with $V \not \subset \mathbf{B}_{+}(L)$.

It is easy to verify that $\operatorname{Psef}_{V}(X)$ is closed and $\operatorname{Big}_{V}(X)$ is its interior. Note also that $\left.L\right|_{V}$ is pseudoeffective whenever $L$ has numerical class in $\operatorname{Psef}_{V}(X)$. The following perspective will sometimes be useful:

Definition 2.7. Suppose that $V \subset X$ is a subvariety. If $L$ is an effective divisor such that $\operatorname{Supp}(L) \not \supset V$, we say $L \geq_{V} 0$.

The relationship with the earlier criteria is given by a trivial lemma.

Lemma 2.8. Suppose that $V \subset X$ is a subvariety. If $L$ is a $V$-big divisor, then $L \sim_{\mathbb{R}} L^{\prime}$ for some $L^{\prime} \geq_{V} 0$.

2C. Admissible and $V$-birational models. Suppose that $X$ is a normal variety and $V$ is a subvariety. In order to study how $V$-pseudoeffective divisors behave under birational pullbacks, we need to be careful about how $V$ intersects the exceptional centers of the map. The most general situation is the following:

Definition 2.9. Let $X$ be a normal variety and $V$ a subvariety of $X$. Suppose that $\phi: Y \rightarrow X$ is a birational map and that $W$ is a subvariety of $Y$ such that the induced map $\left.\phi\right|_{W}: W \rightarrow V$ is generically finite. We say that $(Y, W)$ or $\phi:(Y, W) \rightarrow(X, V)$ is an admissible model for $(X, V)$. When both $Y$ and $W$ are smooth, we say that $(Y, W)$ is a smooth admissible model.

The disadvantage of admissible models is that in many circumstances we need to keep track of the degree of $\left.\phi\right|_{W}$. Since we want to focus on the birational geometry of $V$, we will usually restrict ourselves to the following situation:

Definition 2.10. Let $X$ be a normal variety and $V$ a subvariety not contained in $\operatorname{Sing}(X)$. Suppose that $\phi: \widetilde{X} \rightarrow X$ is a birational map from a normal variety $\widetilde{X}$ such that $V$ is not contained in any $\phi$-exceptional center. Let $\widetilde{V}$ denote the strict transform of $V$. We say that $(\widetilde{X}, \widetilde{V})$ or $\phi: \widetilde{X} \rightarrow X$ is a $V$-birational model for $(X, V)$. When both $\widetilde{X}$ and $\widetilde{V}$ are smooth, we say that $(\tilde{X}, \widetilde{V})$ is a smooth $V$-birational model.

Suppose that $V$ is a subvariety not contained in $\operatorname{Sing}(X)$ and $\phi:(Y, W) \rightarrow(X, V)$ is an admissible model. By Proposition 2.5, the pullback of a $V$-pseudoeffective divisor under $\phi$ is $W$-pseudoeffective. If $\phi$ is a $V$-birational model, then more is true.

Proposition 2.11. Let $X$ be a normal variety and $V$ a subvariety not contained in $\operatorname{Sing}(X)$. Suppose that $\phi: \widetilde{X} \rightarrow X$ is a $V$-birational model. If $L$ is a $V$-big divisor, then $\phi^{*} L$ is a $\widetilde{V}$-big divisor. 
Proof. The $V$-pseudoeffectiveness of $L$ implies that $\phi^{*} L$ is $\widetilde{V}$-pseudoeffective. By openness of the $\widetilde{V}$-big cone, it suffices to check that $\phi^{*} H$ is $\widetilde{V}$-big for an ample divisor $H$ on $X$. Let $\psi: \widetilde{Y} \rightarrow \widetilde{X}$ be a smooth model such that $\psi$ is an isomorphism away from $\operatorname{Sing}(\tilde{X})$. Note that for some sufficiently small $\epsilon$,

$$
\begin{aligned}
\psi^{-1} \mathbf{B}_{+}\left(\phi^{*} H\right) & =\psi^{-1} \mathbf{B}_{\mathbb{R}}\left((1-\epsilon) \phi^{*} H\right) \quad \text { by [Ein et al. 2006, Corollary 1.6] } \\
& =\mathbf{B}_{\mathbb{R}}\left((1-\epsilon) \psi^{*} \phi^{*} H\right) \\
& \subset \mathbf{B}_{+}\left(\psi^{*} \phi^{*} H\right)
\end{aligned}
$$

But clearly $\mathbf{B}_{+}\left(\psi^{*} \phi^{*} H\right)$ is contained in the $(\phi \circ \psi)$-exceptional locus. Thus, $\mathbf{B}_{+}\left(\phi^{*} H\right)$ is contained inside the union of the $\phi$-exceptional locus and $\operatorname{Sing}(\tilde{X})$. In particular, it does not contain $\widetilde{V}$.

2D. Restricted volume. Just as the volume measures the asymptotic rate of growth of sections, the restricted volume measures the rate of growth of restrictions of sections to a subvariety $V$. This notion originated in the work of Hacon-McKernan and Takayama and is systematically developed in [Ein et al. 2009].

Definition 2.12. Suppose that $X$ is a normal variety, $V$ is a $d$-dimensional subvariety of $X$, and $L$ is a divisor. We define

$$
H^{0}\left(X \mid V, \mathscr{O}_{X}(\lfloor L\rfloor)\right):=\operatorname{Im}\left(H^{0}\left(X, \mathscr{O}_{X}(\lfloor m L\rfloor)\right) \rightarrow H^{0}\left(V, \mathscr{O}_{V}(\lfloor m L\rfloor)\right)\right)
$$

and $h^{0}\left(X \mid V, \mathscr{O}_{X}(\lfloor L\rfloor)\right)$ to be the dimension of this space. We then define the restricted volume $\operatorname{vol}_{X \mid V}(L)$ to be

$$
\operatorname{vol}_{X \mid V}(L):=\limsup _{m \rightarrow \infty} \frac{h^{0}\left(X \mid V, \mathcal{O}_{X}(\lfloor m L\rfloor)\right)}{m^{d} / d !} .
$$

Remark 2.13. Although this definition of $\operatorname{vol}_{X \mid V}$ is formulated differently from that of [Ein et al. 2009], the two definitions agree (whenever the restricted volume is defined in [Ein et al. 2009]). An elementary argument proves that $\operatorname{vol}_{X \mid V}$ is homogeneous of degree $d$ so that Definition 2.12 agrees with the definition in [Ein et al. 2009] for $\mathbb{Q}$-divisors. In particular, $\operatorname{vol}_{X \mid V}$ is a continuous function on the space of $V$-big $\mathbb{Q}$-divisors. Using this fact, one readily checks that $\operatorname{vol}_{X \mid V}$ is continuous on the set of $V$-big $\mathbb{R}$-divisors by perturbing by ample divisors and thus coincides with the definition of [Ein et al. 2009].

As with the other quantities we consider, the restricted volume is a numerical and birational invariant. More precisely, [Ein et al. 2009, Theorem A] shows that if $L$ and $L^{\prime}$ are numerically equivalent $V$-big divisors, then $\operatorname{vol}_{X \mid V}(L)=\operatorname{vol}_{X \mid V}\left(L^{\prime}\right)$. Furthermore, [Ein et al. 2009, Proposition 2.4] proves that the restricted volume remains unchanged upon pulling back to an admissible model. 
2E. Twisted linear series. It was observed by litaka that linear series of the form $\lfloor\lfloor m L\rfloor+A \mid$ play an important role in governing the numerical behavior of $L$. Due to the presence of the auxiliary divisor $A$, we call these "twisted" linear series. In this section, we recall the work of Nakayama [2004] analyzing the asymptotic behavior of twisted linear series.

Definition 2.14. Let $X$ be a normal variety, $L$ a pseudoeffective $\mathbb{R}$-divisor, and $A$ any divisor. If $H^{0}\left(X, \mathcal{O}_{X}(\lfloor m L+A\rfloor)\right)$ is nonzero for infinitely many values of $m$, we define

$$
\kappa_{\sigma}(L ; A):=\max \left\{k \in \mathbb{Z}_{\geq 0} \mid \limsup _{m \rightarrow \infty} \frac{h^{0}\left(X, \mathscr{O}_{X}(\lfloor m L+A\rfloor)\right)}{m^{k}}>0\right\} .
$$

Otherwise, we define $\kappa_{\sigma}(L ; A)=-\infty$. The $\sigma$-dimension $\kappa_{\sigma}(X, L)$ is defined to be

$$
\kappa_{\sigma}(L):=\max _{A}\left\{\kappa_{\sigma}(L ; A)\right\} .
$$

Note that this maximum will be computed by some sufficiently ample divisor $A$. Thus, we restrict our attention to the case when $A$ is an ample $\mathbb{Z}$-divisor from now on.

Remark 2.15. As we increase $m$, the class of the divisor $\lceil m L\rceil-\lfloor m L\rfloor$ is bounded. Thus, if we replace $\lfloor-\rfloor$ by $\lceil-\rceil$ in the definition of $\kappa_{\sigma}(L)$, the result is unchanged as the difference can be absorbed by the divisor $A$.

Remark 2.16. Nakayama asks whether $\kappa_{\sigma}(L)$ coincides with

- $\kappa_{\sigma}^{-}(L)$, where we replace the lim sup by a liminf, and

- $\kappa_{\sigma}^{+}(L)$, where we replace $>0$ by $<\infty$.

The equality of these three notions is a consequence of Theorem 6.7(7).

Nakayama shows that $\kappa_{\sigma}$ is a birational and numerical invariant. In fact, since $\kappa_{\sigma}$ is one of the many equivalent definitions of the numerical dimension, it satisfies all of the properties of Theorem 6.7. The following key result shows that $\kappa_{\sigma}$ is nonnegative for pseudoeffective divisors:

Proposition 2.17 [Nakayama 2004, Corollary V.1.4]. Let X be a smooth variety of dimension n. Fix a big basepoint-free divisor $B$ on $X$. Then a divisor $L$ is pseudoeffective if and only if $h^{0}\left(X, \mathcal{O}_{X}\left(K_{X}+(n+2) B+\lceil m L\rceil\right)\right)>0$ for every $m \geq 0$.

Proof. Nakayama's Corollary V.1.4 is actually a similar statement for $B$ very ample. We explain how to extend the argument to the case when $B$ is big and basepoint-free. The main point is to show that there is an effective divisor $D \equiv(n+1) B+\lceil m L\rceil$ such that $\mathscr{f}(D)$ has an isolated point. There is an effective divisor $E \equiv B+\lceil m L\rceil$. Choose a general point $x$ that does not lie in $\operatorname{Supp}(E) \cup \mathbf{B}_{+}(B)$. Let $B_{1}, \ldots, B_{n^{2}} \in|B|$ be irreducible smooth divisors going through $x$. Since $B$ is big, by choosing the $B_{i}$ sufficiently general, we may ensure the intersections of any collection of at most $n$ 
of them has the expected dimension. Thus, $D:=\sum \frac{1}{n} B_{i}+E$ has multiplicity $n$ at $x$ and less than 1 in a neighborhood of $x$. By [Lazarsfeld 2004, Propositions 9.3.2 and 9.5.13], $\mathscr{f}(D)$ has an isolated point. The proof then proceeds as in [Nakayama 2004, Corollary V.1.4].

\section{Divisorial Zariski decomposition}

The divisorial Zariski decomposition is a higher dimension analogue of the classical Zariski decomposition on surfaces. It was introduced by Nakayama [2004] and by Boucksom [2004] in the analytic setting.

Definition 3.1. Let $X$ be a smooth variety, and let $L$ be a pseudoeffective divisor. Fix an ample divisor $A$ on $X$. For any prime divisor $\Gamma$ on $X$, we define

$$
\sigma_{\Gamma}(L)=\lim _{\epsilon \rightarrow 0^{+}} \inf \left\{\operatorname{mult}_{\Gamma}\left(L^{\prime}\right) \mid L^{\prime} \sim_{\mathbb{R}} L+\epsilon A \text { and } L^{\prime} \geq 0\right\} .
$$

By Lemma III.1.5 of [Nakayama 2004], this is independent of the choice of $A$.

Lemma III.1.7 of the same reference says that for any pseudoeffective divisor $L$ there are only finitely many prime divisors $\Gamma$ with $\sigma_{\Gamma}(L)>0$. Thus, we can define the following:

Definition 3.2. Let $X$ be a smooth variety and $L$ a pseudoeffective divisor. Define

$$
N_{\sigma}(L)=\sum \sigma_{\Gamma}(L) \Gamma \quad \text { and } \quad P_{\sigma}(L)=L-N_{\sigma}(L) .
$$

The decomposition $L=N_{\sigma}(L)+P_{\sigma}(L)$ is called the divisorial Zariski decomposition of $L$.

The following proposition records the basic properties of the divisorial Zariski decomposition. The key point is that $P_{\sigma}(L)$ captures all of the interesting geometric information about $L$.

Proposition 3.3 [Nakayama 2004, Lemma III.1.4, Corollary III.1.9, Theorem V.1.3]. Let $X$ be a smooth variety and $L$ a pseudoeffective divisor. Then

(1) $N_{\sigma}(L)$ depends only on the numerical class of $L$,

(2) $N_{\sigma}(L) \geq 0$ and $\kappa\left(N_{\sigma}(L)\right)=0$,

(3) $\operatorname{Supp}\left(N_{\sigma}(L)\right)$ is precisely the divisorial part of $\mathbf{B}_{-}(L)$, and

(4) $H^{0}\left(X, \mathscr{O}_{X}\left(\left\lfloor m P_{\sigma}(L)\right\rfloor\right)\right) \rightarrow H^{0}\left(X, \mathscr{O}_{X}(\lfloor m L\rfloor)\right)$ is an isomorphism for all $m \geq 0$.

Note that $N_{\sigma}(L)=0$ if and only if $\mathbf{B}_{-}(L)$ has no divisorial components. This simple observation leads to a different perspective on the divisorial Zariski decomposition. 
Definition 3.4. Let $X$ be a smooth variety. The movable cone $\overline{M o v}^{1}(X) \subset C D(X)$ is the cone consisting of the classes of all pseudoeffective divisors $L$ such that $\mathbf{B}_{-}(L)$ has no divisorial components.

The positive part $P_{\sigma}(L)$ of the divisorial Zariski decomposition can be understood as a "projection" of $L$ onto the movable cone. We will need a slightly modified version of [Nakayama 2004, Proposition III.1.14] that takes into account a subvariety $V$.

Proposition 3.5. Let $X$ be smooth, $V$ a subvariety, and $L$ a $V$-pseudoeffective divisor. If $M$ is a movable divisor, then $L \geq_{V} M$ if and only if $P_{\sigma}(L) \geq_{V} M$. Thus, $L-M$ is $V$-big or $V$-pseudoeffective if and only if $P_{\sigma}(L)-M$ is $V$-big or $V$-pseudoeffective, respectively.

Proof. First suppose that $P_{\sigma}(L) \geq_{V} M$. Since $L$ is $V$-pseudoeffective, no component of $N_{\sigma}(L)$ contains $V$. Thus, $L \geq_{V} M$. Conversely, suppose $L=M+E$ with $E \geq_{V} 0$. Since $M$ is movable, $N_{\sigma}(L) \leq E$ by [Nakayama 2004, Proposition III.1.14]. Thus, $E-N_{\sigma}(L)$ is still effective and does not contain $V$ in its support, showing that $P_{\sigma}(L) \geq_{V} M$.

Suppose now that $L-M$ is $V$-big. Choose an ample divisor $A$ sufficiently small so that $L-M-A$ is $V$-big. By Lemma 2.8, there is some $D \sim_{\mathbb{R}} L-M-A$ such that $D \geq_{V} 0$. Applying the first step to $L-D$ shows that $P_{\sigma}(L)-L+D \equiv P_{\sigma}-M-A$ is $V$-pseudoeffective so that $P_{\sigma}(L)-M$ is $V$-big. The converse is straightforward. The analogous statement for $V$-pseudoeffectiveness follows by taking limits.

3A. Birational properties. Although the divisorial Zariski decomposition is not a birational invariant, its birational behavior is relatively nice.

Proposition 3.6 [Nakayama 2004, Theorem III.5.16]. Let $\phi: Y \rightarrow X$ be a birational map of smooth varieties, and let $L$ be a pseudoeffective divisor on $X$. Then $N_{\sigma}\left(\phi^{*} L\right)-\phi^{*} N_{\sigma}(L)$ is effective and $\phi$-exceptional.

We say $L$ admits a Zariski decomposition if there is a birational map $\phi: Y \rightarrow X$ from a smooth variety $Y$ such that $P_{\sigma}\left(\phi^{*} L\right)$ is numerically effective. An important example due to Nakayama [2004, Section IV.2] shows that Zariski decompositions do not always exist. Nevertheless, there is a sense in which the positive part $P_{\sigma}\left(\phi^{*} L\right)$ becomes "more numerically effective" as we pass to higher models $\phi: Y \rightarrow X$. We will give two versions of this fact. In the first, we consider a $V$-big divisor $L$.

Proposition 3.7. Let $X$ be smooth, $V$ a subvariety, and $L$ a $V$-big divisor with $L \geq_{V} 0$. Then there is an effective divisor $G$ so that for any sufficiently large $m$ there is a model $\phi_{m}: \widetilde{X}_{m} \rightarrow X$ centered in $\mathbf{B}_{+}(L)$ and a big and numerically effective divisor $N_{m}$ on $\widetilde{X}_{m}$ such that, with $\widetilde{V}_{m}$ denoting the strict transform of $V$ on $\widetilde{X}_{m}$,

$$
N_{m} \leq \widetilde{V}_{m} P_{\sigma}\left(\phi_{m}^{*} L\right) \leq \widetilde{V}_{m} N_{m}+\frac{1}{m} \phi_{m}^{*} G .
$$


The second version handles $V$-pseudoeffective divisors $L$. Although the statement is slightly more technical, the additional flexibility will be useful later on.

Proposition 3.8. Let $X$ be smooth, and let $L$ be a pseudoeffective divisor. There are birational maps $\phi_{m}: \widetilde{X}_{m} \rightarrow X$ centered in $\mathbf{B}_{-}(L)$, an ample $\mathbb{Z}$-divisor $A$, and an effective divisor $G$ satisfying the following condition. Suppose that $V$ is a subvariety of $X$ not contained in $\mathbf{B}_{-}(L)$. Then there is some $G_{V} \sim_{\mathbb{Q}} G$, and for every $m$, there is an effective divisor $D_{m} \sim\lceil m L\rceil+A$ and a big and numerically effective divisor $M_{m, D_{m}}$ such that

$$
M_{m, D_{m}} \leq \widetilde{V}_{m} P_{\sigma}\left(\phi_{m}^{*} D_{m}\right) \leq_{\widetilde{V}_{m}} M_{m, D_{m}}+\phi_{m}^{*} G_{V},
$$

where $\widetilde{V}_{m}$ denotes the strict transform of $V$ on $\widetilde{X}_{m}$. We may furthermore assume that $A+D$ is ample for every $D$ supported on $\operatorname{Supp}(L)$ with coefficients in the set $[-3,3]$.

Proposition 3.7 is equivalent to the following comparison between asymptotic multiplier ideals and base loci. It is the analogue for $\mathbb{R}$-divisors of [Lazarsfeld 2004, Theorem 11.2.21]. Note that the theory of asymptotic multiplier ideals for big $\mathbb{R}$-divisors works just as in the case of $\mathbb{Q}$-divisors.

Lemma 3.9. Let $X$ be smooth, and let $L$ be a big divisor on $X$. Fix a very ample $\mathbb{Z}$ divisor $H$ on $X$ such that $H+D$ is ample for every divisor $D$ supported on $\operatorname{Supp}(L)$ with coefficients in the set $[-3,3]$. Suppose that $b$ is a sufficiently large positive integer so that $\lfloor b L\rfloor-\left(K_{X}+(n+1) H\right)$ is numerically equivalent to an effective $\mathbb{Z}$-divisor $G$. Then for every $m \geq b$, we have

$$
\mathscr{f}(\|m L\|) \otimes \mathcal{O}_{X}(-G) \subseteq \mathfrak{b}(|\lfloor m L\rfloor|) .
$$

Proof. The condition on $H$ guarantees that for $m \geq b$, we can write

$$
\begin{aligned}
\lfloor m L\rfloor-G & \equiv\lfloor m L\rfloor-\lfloor b L\rfloor+K_{X}+(n+1) H \\
& \equiv((m-b) L+A)+K_{X}+n H
\end{aligned}
$$

for some ample $\mathbb{R}$-divisor $A$. By applying Nadel vanishing and CastelnuovoMumford regularity, we find that

$$
\mathcal{O}_{X}(\lfloor m L\rfloor) \otimes\left(\mathscr{O}_{X}(-G) \otimes \mathscr{g}(\|(m-b) L\|)\right)
$$

is globally generated for $m \geq b$. Then $\mathscr{F}(\|m L\|) \subset \mathscr{G}(\|(m-b) L\|)$.

Proof of Proposition 3.7. Fix a very ample $\mathbb{Z}$-divisor $H$ and an integer $b$ as in Lemma 3.9. Thus, for any $m \geq b$, we have

$$
\mathscr{f}(\|m L\|) \otimes \mathcal{O}_{X}(-G) \subseteq \mathfrak{b}(|\lfloor m L\rfloor|) .
$$

Recall that $G$ can be chosen to be any effective $\mathbb{Z}$-divisor numerically equivalent to $\lfloor b L\rfloor-\left(K_{X}+(n+1) H\right)$. In particular, for $b$ large enough, the base locus of $|G|$ is contained in $\mathbf{B}_{+}(L)$. Since this set does not contain $V$, we may ensure that $G \geq_{V} 0$. 
Let $\phi_{m}: \widetilde{X}_{m} \rightarrow X$ be a resolution of the ideals $\mathfrak{b}(|\lfloor m L\rfloor|)$ and $\mathscr{\mathscr { L }}(\|m L\|)$. Note that each $\phi_{m}$ is centered in $\mathbf{B}_{+}(L)$. We write $\phi_{m}^{-1} \mathfrak{b}(|\lfloor m L\rfloor|) \cdot \mathscr{O}_{Y_{m}}=\mathcal{O}_{Y_{m}}\left(-E_{m}\right)$ and $\phi_{m}^{-1} \mathscr{S}(\|m L\|) \cdot \mathrm{O}_{Y_{m}}=\mathrm{O}_{Y_{m}}\left(-F_{m}\right)$. We also define the big and numerically effective divisor $M_{m}:=m \phi_{m}^{*} L-E_{m}-\phi_{m}^{*}\{m L\}$.

We know that $F_{m}+\phi_{m}^{*} G \geq E_{m}$ for all sufficiently large $m$. Let $M=\sum_{D \subset \operatorname{Supp}(L)} D$ be the sum of the components of $\operatorname{Supp}(L)$. Replacing $G$ by $G+M$ allows us to take into account the fractional part of $m L$ so that

$$
F_{m}+\phi_{m}^{*} G \geq E_{m}+\phi_{m}^{*}\{m L\} .
$$

Note that still $G \geq_{V} 0$. Since $L$ is $V$-big, we know that $F_{m} \geq \widetilde{V}_{m} 0$. Thus, the inequality in the equation above is a $\widetilde{V}_{m}$-inequality. Furthermore, $N_{\sigma}\left(m \phi_{m}^{*} L\right) \geq \widetilde{V}_{m} F_{m}$ by [Ein et al. 2006, Proposition 2.5]. In all, we get $P_{\sigma}\left(m \phi_{m}^{*} L\right) \leq \widetilde{V}_{m} M_{m}+\phi_{m}^{*} G$. Dividing by $m$ and setting $N_{m}:=M_{m} / m$ yields $P_{\sigma}\left(\phi_{m}^{*} L\right) \leq \widetilde{V}_{m} N_{m}+\frac{1}{m} \phi_{m}^{*} G$. The inequality $N_{m} \leq \widetilde{V}_{m} P_{\sigma}\left(\phi_{m}^{*} L\right)$ follows from Proposition 3.5 and the fact that $E_{m}+\phi_{m}^{*}\{m L\} \geq \widetilde{V}_{m} 0$.

Proof of Proposition 3.8. Fix very ample divisors $H$ and $G$. By Theorem 2.4, there is an ample $\mathbb{Z}$-divisor $A$ such that $\operatorname{Bs}(|\lceil m L\rceil+A|) \subset \mathbf{B}_{-}(L)$ for every positive integer $m$. We may assume that $A$ is sufficiently ample so that

- $\lceil m L\rceil+A-K_{X}-(n+1) H$ is numerically equivalent to an effective divisor $G_{m}$ for every $m>0$ and

- $A+D$ is ample for every $D$ supported on $\operatorname{Supp}(L)$ with coefficients in $[-3,3]$. Choose $D_{m} \sim\lceil m L\rceil+A$ so that $D_{m} \geq_{V} 0$. Note that we can apply Proposition 3.7 to $D_{m}$ using $G_{m}$ as our choice of effective divisor (since $D_{m}$ is an integral divisor, there is no need to set conditions on the ampleness of $H$ along the components of $D_{m}$ ). In particular, for every positive integer $m$, choose an $\epsilon_{m}>0$ such that $G-\epsilon_{m} G_{m}$ is ample. Proposition 3.7 constructs a birational map $\phi_{m}: X_{m} \rightarrow X$ and big and numerically effective divisors $M_{m, D_{m}}$ such that

$$
M_{m, D_{m}} \leq \widetilde{V}_{m} P_{\sigma}\left(\phi_{m}^{*} D_{m}\right) \leq \widetilde{V}_{m} M_{m, D_{m}}+\epsilon_{m} \phi_{m}^{*} G_{m} .
$$

Since $G-\epsilon_{m} G_{m}$ is $V$-big, we may replace $G$ by some $\mathbb{Q}$-linearly equivalent divisor $G_{V}$ so that

$$
M_{m, D_{m}} \leq \widetilde{V}_{m} P_{\sigma}\left(\phi_{m}^{*} D_{m}\right) \leq_{\widetilde{V}_{m}} M_{m, D_{m}}+\phi_{m}^{*} G_{V}
$$

\section{The restricted positive product}

Fujita realized that one can study the asymptotic behavior of sections of a big divisor $L$ by analyzing the ample divisors sitting beneath $L$ on higher birational models. The positive product (developed in [Boucksom 2004; Boucksom et al. 2012]) is a construction that encapsulates this approach to asymptotic behavior. 
In this section, we discuss the restricted positive product $\left\langle L_{1} \cdot L_{2} \cdots L_{k}\right\rangle_{X \mid V}$ of Boucksom, Favre, and Jonsson [Boucksom et al. 2009]. Unlike the usual intersection product $L_{1} \cdot L_{2} \cdots \cdot L_{k} \cdot V$, the restricted positive product throws away the contributions of the base loci of the $L_{i}$. The result is a numerical equivalence class of cycles on $V$ that gives a more precise measure of the positivity of the $L_{i}$ along $V$.

4A. Definition and basic properties. We start by reviewing the construction of the restricted positive product in [Boucksom et al. 2009]. Throughout, we will use the intersection product of [Fulton 1984]. We will use the following notation:

Definition 4.1. Let $X$ be a normal variety. Suppose that $V$ is a subvariety of $X$ and that $[L] \in C D(X)$. We will let $\left.[L]\right|_{V}$ denote the image under the restriction map $C D^{1}(X) \rightarrow C D^{1}(V)$.

Note that if $L$ is a divisor such that $\operatorname{Supp}(L) \not \supset V$, then $\left[\left.L\right|_{V}\right]=\left.[L]\right|_{V}$.

Definition 4.2. Let $X$ be a normal variety of dimension $n$. Suppose that $K$ and $K^{\prime}$ are two classes in $N^{k}(X)$. We write $K \succeq K^{\prime}$ if $K-K^{\prime}$ is contained in the closure of the cone generated by effective cycles of dimension $n-k$.

Lemma 4.3 [ibid., Proposition 2.3, Definition 4.4]. Let $X$ be a smooth variety and $V$ a subvariety of $X$. Suppose that $N_{1}, \ldots, N_{k}$ and $N_{1}^{\prime}, \ldots, N_{k}^{\prime}$ are numerically effective divisors on $X$ satisfying $N_{i} \geq_{V} N_{i}^{\prime}$. Then

$$
N_{1} \cdots \cdot N_{k} \cdot V \succeq N_{1}^{\prime} \cdots \cdot N_{k}^{\prime} \cdot V .
$$

Theorem 4.4 [ibid., Lemmas 2.6 and 2.7]. Let $X$ be a normal variety, $V$ a subvariety not contained in $\operatorname{Sing}(X)$, and $L_{1}, \ldots, L_{k} V$-big divisors. Consider the classes

$$
\phi_{*}\left(N_{1} \cdot N_{2} \cdots \cdot N_{k} \cdot \widetilde{V}\right) \in N^{k}(V),
$$

where $\phi:(\tilde{X}, \tilde{V}) \rightarrow(X, V)$ varies over all smooth $V$-birational models, the $N_{i}$ are numerically effective, and $E_{i}:=\phi^{*} L_{i}-N_{i}$ is a $\mathbb{Q}$-divisor satisfying $E_{i} \geq \widetilde{V} 0$. These classes form a directed set under the relation $\preceq$ and admit a unique maximum under this relation.

Remark 4.5. Although [Boucksom et al. 2009] only proves this when $V$ is a prime divisor in $X$, the proof works without change in this more general situation.

The restricted positive product is defined as the maximum class occurring in the previous theorem.

Definition 4.6. Let $X$ be a normal variety, and let $V$ be a subvariety not contained in $\operatorname{Sing}(X)$. Let $L_{1}, L_{2}, \ldots, L_{k}$ be $V$-big divisors. We define the cycle

$$
\left\langle L_{1} \cdot L_{2} \cdots \cdot L_{k}\right\rangle_{X \mid V} \in N^{k}(V)
$$

as the maximum under $\preceq$ of $\phi_{*}\left(N_{1} \cdot N_{2} \cdots \cdots N_{k} \cdot \widetilde{V}\right)$, where $\phi:(\tilde{X}, \widetilde{V}) \rightarrow(X, V)$ 
runs over smooth $V$-birational models, the $N_{i}$ are numerically effective and $E_{i}:=$ $\phi^{*} L_{i}-N_{i}$ is a $\mathbb{Q}$-divisor satisfying $E_{i} \geq \widetilde{V} 0$. In the special case $X=V$, we write $\left\langle L_{1} \cdot L_{2} \cdots \cdot L_{k}\right\rangle_{X}$.

In fact, [ibid., Proposition 2.13] shows that the definition is unchanged if we allow $E_{i}$ to be a $V$-pseudoeffective $\mathbb{R}$-divisor. The restricted positive product satisfies a number of important properties.

Proposition 4.7 [ibid., Proposition 4.6]. As a function on the k-fold product of the $V$-big cone, the restricted positive product is continuous, symmetric, homogeneous of degree 1, and superadditive in each variable in the sense that

$$
\left\langle\left(L+L^{\prime}\right) \cdot L_{2} \cdots \cdot L_{k}\right\rangle_{X \mid V} \succeq\left\langle L \cdot L_{2} \cdots L_{k}\right\rangle_{X \mid V}+\left\langle L^{\prime} \cdot L_{2} \cdots L_{k}\right\rangle_{X \mid V} .
$$

Since the product is continuous, this allows us to define a limit as we approach the pseudoeffective cone.

Definition 4.8. Let $X$ be a normal variety, $V$ a subvariety not contained in $\operatorname{Sing}(X)$, and $L_{1}, L_{2}, \ldots, L_{k} V$-pseudoeffective divisors. For each $i$, fix a sequence of $V$-big divisors $B_{i, j}$ converging to 0 as $j$ increases. We define the class

$$
\left\langle L_{1} \cdot L_{2} \cdots \cdot L_{k}\right\rangle_{X \mid V}=\lim _{j \rightarrow \infty}\left\langle\left(L_{1}+B_{1, j}\right) \cdot\left(L_{2}+B_{2, j}\right) \cdots \cdot\left(L_{k}+B_{k, j}\right)\right\rangle_{X \mid V} \cdot
$$

Note that this limit is independent of the choice of the $B_{i, j}$ since by superadditivity any two choices are comparable under $\succeq$.

We will sometimes abuse notation by allowing the restricted positive product to take numerical classes as arguments rather than actual divisors. Since the restricted positive product is compatible under pushforward, we can extend the definition to arbitrarily singular varieties in the following way:

Definition 4.9. Let $X$ be an integral variety, and let $\phi: Y \rightarrow X$ be a smooth model. For $\left[L_{1}\right], \ldots,\left[L_{k}\right] \in C D(X)$, we define

$$
\left\langle\left[L_{1}\right] \cdots \cdot\left[L_{k}\right]\right\rangle_{X}:=\phi_{*}\left\langle\phi^{*}\left[L_{1}\right] \cdots \cdot \phi^{*}\left[L_{k}\right]\right\rangle_{Y} .
$$

Even though the restricted positive product is continuous along the $V$-big cone, it is only semicontinuous along the $V$-pseudoeffective boundary in the sense that if $L_{i, j}$ is a sequence of $V$-pseudoeffective divisors whose limit is $L_{i}$, then

$$
\left\langle L_{1} \cdots L_{k}\right\rangle_{X \mid V} \succeq \lim _{j \rightarrow \infty}\left\langle L_{1, j} \cdots \cdot L_{k, j}\right\rangle_{X \mid V} .
$$

As noted in [Boucksom et al. 2009], it is most natural to consider the restricted positive product as the set of classes $\left\{\left\langle\phi^{*} L_{1} \cdots \cdot \phi^{*} L_{k}\right\rangle_{\tilde{X} \mid \tilde{V}}\right\}$ on all smooth $V$ birational models $\phi: \widetilde{X} \rightarrow X$ or, in other words, as a class on the Riemann-Zariski space of $V$. Although we will not develop this principle systematically, this idea 
appears implicitly as some theorems will only hold upon taking a limit over all sufficiently high birational models.

Since the restricted positive product should be considered as a birational object, the class in $N^{k}(V)$ may not be closely related to the geometry of $L$ and $V$. The class $\left\langle L_{1} \cdots L_{k}\right\rangle_{X \mid V}$ seems to be most interesting in the following two situations:

Example 4.10. When $X$ is smooth, $\langle L\rangle_{X}$ is the numerical class of $P_{\sigma}(L)$. It suffices to check this when $L$ is big. Recall that for any birational map $\phi: Y \rightarrow X$ from a smooth variety $Y$, we have $\phi_{*} P_{\sigma}\left(\phi^{*} L\right)=P_{\sigma}(L)$. Thus, choosing an effective divisor $G$ as in Proposition 3.7, the result of the proposition implies that for any $\epsilon>0$, we have $\langle L\rangle_{X} \preceq\left[P_{\sigma}(L)\right] \preceq\langle L+\epsilon G\rangle_{X}$. Letting $\epsilon \rightarrow 0$ demonstrates the equality.

Example 4.11. Consider $\left\langle L_{1} \cdots L_{d}\right\rangle_{X \mid V}$, where $d=\operatorname{dim} V$. Since the restricted positive product is compatible under pushforward, $\operatorname{deg}\left\langle\phi^{*} L_{1} \cdots \cdot \phi^{*} L_{d}\right\rangle_{\tilde{X} \mid \tilde{V}}$ is independent of the choice of $V$-birational model $(\tilde{X}, \widetilde{V})$ by the projection formula. In fact, we have the following:

Proposition 4.12 [Ein et al. 2009, Proposition 2.11, Theorem 2.13]. Let $X$ be a smooth variety, $V$ a d-dimensional subvariety, and $L$ a $V$-big divisor. Then $\operatorname{deg}\left\langle L^{d}\right\rangle_{X \mid V}=\operatorname{vol}_{X \mid V}(L)$.

4B. Properties of the restricted positive product. In this section, we study the properties of the restricted positive product. The main goal of the section is to show that the restricted positive product can be interpreted as the usual intersection product of $P_{\sigma}\left(\phi^{*} L_{i}\right)$ if we take a limit over all birational models $\phi$. The advantage of this viewpoint is that it gives us a natural interpretation of the restricted positive product along the boundary of the pseudoeffective cone.

We first show that the restricted positive product has a natural compatibility with the divisorial Zariski decomposition.

Proposition 4.13. Let $X$ be a smooth variety, $V$ a subvariety, and $L_{1}, \ldots, L_{k}$ $V$-pseudoeffective divisors. Then

$$
\left\langle L_{1} \cdots L_{k}\right\rangle_{X \mid V}=\left\langle P_{\sigma}\left(L_{1}\right) \cdots \cdot P_{\sigma}\left(L_{k}\right)\right\rangle_{X \mid V} .
$$

Proof. First suppose that the $L_{i}$ are $V$-big. Since any numerically effective divisor is movable, Proposition 3.5 shows that for any of the $N_{i}$ as in Definition 4.6, we have $P_{\sigma}\left(\phi^{*} L_{i}\right) \geq \widetilde{V} N_{i}$. We also know that $N_{\sigma}\left(\phi^{*} L_{i}\right) \geq \widetilde{V} \phi^{*} N_{\sigma}\left(L_{i}\right)$ since $V$ is not contained in $\mathbf{B}_{-}\left(L_{i}\right)$. Combining the two inequalities yields

$$
\phi^{*} P_{\sigma}\left(L_{i}\right) \geq \widetilde{V} N_{i} .
$$

Thus, the classes $\left\langle L_{1} \cdots L_{k}\right\rangle_{X \mid V}$ and $\left\langle P_{\sigma}\left(L_{1}\right) \cdots P_{\sigma}\left(L_{k}\right)\right\rangle_{X \mid V}$ are computed by taking a maximum over the same sets, showing that they are equal. 
Now suppose that the $L_{i}$ are only $V$-pseudoeffective. Fix an ample divisor $A$ on $X$. Note that

$$
P_{\sigma}(L+\epsilon A)-P_{\sigma}(L)=\epsilon A+\left(N_{\sigma}(L)-N_{\sigma}(L+\epsilon A)\right)
$$

is $V$-big. As $\epsilon$ goes to 0 , these $V$-big classes also converge to 0 . Thus,

$$
\left\langle P_{\sigma}\left(L_{1}\right) \cdots P_{\sigma}\left(L_{k}\right)\right\rangle_{X \mid V}=\lim _{\epsilon \rightarrow 0}\left\langle P_{\sigma}\left(L_{1}+\epsilon A\right) \cdots P_{\sigma}\left(L_{k}+\epsilon A\right)\right\rangle_{X \mid V} .
$$

Applying the $V$-big case to the right-hand side finishes the proof.

The following proposition compares the restricted positive product of the $L_{i}$ along $V$ with the positive product of the restrictions $\left.L_{i}\right|_{V}$. The statement is proved in [Boucksom et al. 2009] only when the $L_{i}$ are $V$-big, but the proposition extends to the $V$-pseudoeffective case by taking limits.

Proposition 4.14 [Boucksom et al. 2009, Remark 4.5]. Let $X$ be a smooth variety, $V$ a subvariety, and $L_{1}, \ldots, L_{k} V$-pseudoeffective divisors. Then

$$
\left\langle L_{1} \cdots \cdot L_{k}\right\rangle_{X \mid V} \preceq\left\langle\left.\left.\left[L_{1}\right]\right|_{V} \cdots \cdot\left[L_{k}\right]\right|_{V}\right\rangle_{V} .
$$

By combining Propositions 4.13 and 4.14, we obtain

$$
\left\langle L_{1} \cdots \cdot L_{k}\right\rangle_{X \mid V} \preceq \phi_{*}\left\langle\left.\left.\left[P_{\sigma}\left(\phi^{*} L_{1}\right)\right]\right|_{\widetilde{V}} \cdots \cdot\left[P_{\sigma}\left(\phi^{*} L_{k}\right)\right]\right|_{\tilde{V}}\right\rangle_{\tilde{V}},
$$

where $\phi:(\tilde{X}, \widetilde{V}) \rightarrow(X, V)$ is any $V$-birational model. The main theorem of this section states that by taking a limit over all birational models, the right-hand side approaches the left.

Theorem 4.15. Let $X$ be a smooth variety, $V$ a subvariety, and $L_{1}, \ldots, L_{k} V$ pseudoeffective divisors. Fix an ample divisor A. Then for any $\epsilon$, there is some $V$-birational map $\phi:(\widetilde{X}, \widetilde{V}) \rightarrow(X, V)$ such that

$$
\phi_{*}\left\langle\left.\left.\left[P_{\sigma}\left(\phi^{*} L_{1}\right)\right]\right|_{\tilde{V}} \cdots \cdot\left[P_{\sigma}\left(\phi^{*} L_{k}\right)\right]\right|_{\tilde{V}}\right\rangle_{\widetilde{V}} \preceq\left\langle L_{1} \cdots L_{k}\right\rangle_{X \mid V}+\epsilon A^{k} \cdot V .
$$

Proof. First suppose the $L_{i}$ are $V$-big. By Lemma 2.8, we may replace the $L_{i}$ by some $\mathbb{R}$-linearly equivalent divisors to ensure that $L_{i} \geq_{V} 0$. Proposition 3.7 then yields an effective divisor $G_{i}$ such that for any $m$ there is a $V$-birational model $\phi: \widetilde{X}_{m} \rightarrow X$ with

$$
N_{m, i} \leq \widetilde{V} P_{\sigma}\left(\phi_{m}^{*} L_{i}\right) \leq \widetilde{V} N_{m, i}+\frac{1}{m} \phi_{m}^{*} G_{i}
$$

for some numerically effective divisors $N_{m, i}$. Fix some ample divisor $A$ on $X$ such that $A-L_{i}$ and $A-G_{i}$ are ample for every $i$. By Lemma 4.3, there is some constant $C$ such that

$\phi_{m *}\left\langle\left.\left.\left[P_{\sigma}\left(\phi_{m}^{*} L_{1}\right)\right]\right|_{\widetilde{V_{m}}} \cdots\left[P_{\sigma}\left(\phi_{m}^{*} L_{k}\right)\right]\right|_{\widetilde{V_{m}}}\right)_{\widetilde{V_{m}}} \preceq \phi_{*}\left(N_{m, 1} \cdots \cdot N_{m, k} \cdot \widetilde{V}\right)+\frac{C}{m} A^{k} \cdot V$. 
Now suppose that the $L_{i}$ are only $V$-pseudoeffective. We first choose an ample divisor $H$ so that

$$
\left\langle\left(L_{1}+H\right) \cdots\left(L_{k}+H\right)\right\rangle_{X \mid V} \preceq\left\langle L_{1} \cdots \cdot L_{k}\right\rangle_{X \mid V}+\frac{\epsilon}{2} A^{k} \cdot V .
$$

Construct a model $\phi$ by applying the $V$-big case to the $L_{i}+H$ and $\epsilon / 2$. Since $P_{\sigma}\left(\phi^{*}\left(L_{i}+H\right)\right)-P_{\sigma}\left(\phi^{*} L\right)$ is $\widetilde{V}$-pseudoeffective, the conclusion follows.

Corollary 4.16. Let $X$ be a smooth variety, and let $L_{1}, \ldots, L_{k}$ be pseudoeffective divisors. There is a sequence of birational maps $\phi_{m}: X_{m} \rightarrow X$ centered in $\cup_{i} \mathbf{B}_{-}\left(L_{i}\right)$ such that for any subvariety $V$ not contained in $\cup_{i} \mathbf{B}_{-}\left(L_{i}\right)$, we have

$$
\left\langle L_{1} \cdots \cdot L_{k}\right\rangle_{X \mid V}=\lim _{m \rightarrow \infty} \phi_{m *}\left\langle\left.\left.\left[P_{\sigma}\left(\phi_{m}^{*} L_{1}\right)\right]\right|_{\widetilde{V}_{m}} \cdots \cdot\left[P_{\sigma}\left(\phi_{m}^{*} L_{k}\right)\right]\right|_{\widetilde{V}_{m}}\right)_{\widetilde{V}_{m}} \cdot
$$

Proof. Fix a sequence of birational maps $\phi_{m}$, an ample divisor $A$, and an effective divisor $G$ as in Proposition 3.8 for each of the $L_{i}$ simultaneously. The proposition constructs divisors $D_{m, i} \equiv\left\lceil m L_{i}\right\rceil+A$ and big and numerically effective divisors $M_{m, i, D_{m, i}}$ such that

$$
M_{m, i, D_{m, i}} \leq \widetilde{V}_{m} P_{\sigma}\left(\phi_{m}^{*} D_{m, i}\right) \leq \widetilde{V}_{m} M_{m, i, D_{m, i}}+\phi_{m}^{*} G_{V} .
$$

Just as in the previous proposition, we have

$$
\begin{aligned}
\lim _{m \rightarrow \infty} & \frac{1}{m^{k}} \phi_{m *}\left(M_{m, 1, D_{m, 1}} \cdots M_{m, k, D_{m, k}} \cdot \widetilde{V}_{m}\right) \\
& \preceq \lim _{m \rightarrow \infty} \frac{1}{m^{k}}\left\langle D_{m, 1} \cdots D_{m, k}\right\rangle_{X \mid V} \\
& \preceq \lim _{m \rightarrow \infty} \frac{1}{m^{k}} \phi_{m *}\left(\left.\left.\left[P_{\sigma}\left(\phi_{m}^{*} D_{m, 1}\right)\right]\right|_{\widetilde{V}_{m}} \cdots \cdot\left[P_{\sigma}\left(\phi_{m}^{*} D_{m, k}\right)\right]\right|_{\widetilde{V}_{m}}\right)_{\widetilde{V}_{m}} \\
& \preceq \lim _{m \rightarrow \infty} \frac{1}{m^{k}} \phi_{m *}\left(\left(M_{m, 1, D_{m, 1}}+\phi_{m}^{*} G_{V}\right) \cdots\left(M_{m, k, D_{m, k}}+\phi_{m}^{*} G_{V}\right) \cdot \widetilde{V}_{m}\right) .
\end{aligned}
$$

Arguing as in the previous proof, we see that the leftmost and rightmost expressions converge as $m$ increases. Recall that by our choice of $A$ we have $\left\lceil m L_{i}\right\rceil+A-m L_{i}$ is $V$-big for every $m$. Thus,

$$
\left\langle L_{1} \cdots \cdot L_{k}\right\rangle_{X \mid V}=\lim _{m \rightarrow \infty}\left\langle\frac{1}{m} D_{m, 1} \cdots \frac{1}{m} D_{m, k}\right\rangle_{X \mid V}
$$

so that the sequence converges to the restricted positive product as desired.

We extract a useful feature of the previous arguments as a definition.

Definition 4.17. Let $X$ be a smooth variety, $V$ a subvariety, and $L_{1}, \ldots, L_{k} V$-big divisors. Choose $L_{i}^{\prime} \sim_{\mathbb{Q}} L_{i}$ satisfying $L_{i}^{\prime} \geq_{V} 0$. Suppose that $\phi_{m}$ is a countable sequence of maps that satisfy the conclusion of Proposition 3.7 for every $L_{i}^{\prime}$ simultaneously. We say that the $\phi_{m}$ compute the restricted positive product of the $L_{i}$. 
Note that for any finite set of subvarieties $V_{1}, \ldots, V_{r}$, we can choose $\phi_{m}$ and $N_{m}$ to simultaneously compute the restricted positive product for each $V_{j}$. The key property of Definition 4.17 is that only countably many maps are needed to compute the restricted positive product.

The restricted positive product reduces to the usual product for numerically effective divisors.

Lemma 4.18. Let $X$ be a smooth variety, $V$ a subvariety, and $L_{1}, \ldots, L_{k} V$ pseudoeffective divisors.

(1) Suppose $N$ is a numerically effective divisor. Then

$$
\left\langle L_{1} \cdot L_{2} \cdots L_{k} \cdot N\right\rangle_{X \mid V}=\left.\left\langle L_{1} \cdot L_{2} \cdots \cdot L_{k}\right\rangle_{X \mid V} \cdot N\right|_{V} .
$$

(2) If $H$ is a very general element of a basepoint-free linear system, then

$$
\left\langle L_{1} \cdot L_{2} \cdots L_{k}\right\rangle_{X \mid V} \cdot H=\left\langle L_{1} \cdot L_{2} \cdots L_{k}\right\rangle_{X \mid V \cap H} .
$$

(3) If $f: X \rightarrow Z$ is a morphism and $F$ is a very general fiber, then

$$
\left\langle L_{1} \cdot L_{2} \cdots \cdot L_{k}\right\rangle_{X \mid V} \cdot F=\left\langle L_{1} \cdot L_{2} \cdots L_{k}\right\rangle_{X \mid V \cap F} .
$$

Proof. For each of these properties, it is enough to check the case when the $L_{i}$ are $V$-big.

The first property is shown in [Boucksom et al. 2009, Proposition 4.7]; one simply notes that for an ample divisor $A$ the pullback $\phi^{*} A$ is already numerically effective so that one may take $\phi^{*} A$ to be the numerically effective divisor in Definition 4.6. By taking limits as $A$ approaches $N$, we obtain the statement.

To show the second property, consider a countable set of smooth $V$-birational models $\phi_{m}: \widetilde{X}_{m} \rightarrow X$ that compute the restricted positive product. Choose $H$ sufficiently general so that it does not contain any $\phi_{m}$-exceptional center. Then the strict transform of $V \cap H$ is a cycle representing the class $\phi_{m}^{*} H \cdot \widetilde{V}$. Thus, we can identify the classes

$$
\begin{aligned}
\phi_{m *}\left(N_{1} \cdot N_{2} \cdots \cdots N_{k} \cdot \tilde{V}\right) \cdot H & =\phi_{m *}\left(N_{1} \cdot N_{2} \cdots N_{k} \cdot \phi_{m}^{*} H \cdot \tilde{V}\right) \\
& =\phi_{m *}\left(N_{1} \cdot N_{2} \cdots N_{k} \cdot \widetilde{V \cap H}\right) .
\end{aligned}
$$

The third property can be proved by a similar argument. One uses the second property inductively by pulling back very ample divisors from $Z$.

Corollary 4.19. Let $X$ be a normal variety, $V$ a subvariety not contained in $\operatorname{Sing}(X)$, and $L_{1}, \ldots, L_{k} V$-pseudoeffective divisors. Suppose $\phi:(\tilde{X}, \widetilde{V}) \rightarrow(X, V)$ is a smooth $V$-birational model. If $\left\langle\phi^{*} L_{1} \cdots \phi^{*} L_{k}\right\rangle_{\widetilde{X} \mid \widetilde{V}} \neq 0$, then $\left\langle L_{1} \cdots L_{k}\right\rangle_{X \mid V} \neq 0$. 
Proof. Let $A$ be an ample divisor on $\widetilde{X}$, and let $H$ be an ample divisor on $X$ such that $\phi^{*} H \geq A$. Since $\phi$ is $V$-birational, we may ensure that $\operatorname{Supp}\left(\phi^{*} H-A\right)$ does not contain $\widetilde{V}$. Setting $d=\operatorname{dim} V$, we have

$$
\begin{aligned}
\left\langle L_{1} \cdots L_{k}\right\rangle_{X \mid V} \cdot H^{d-k} & =\left\langle\phi^{*} L_{1} \cdots \phi^{*} L_{k}\right\rangle_{\widetilde{X} \mid \widetilde{V}} \cdot \phi^{*} H^{d-k} \\
& =\left\langle\phi^{*} L_{1} \cdots \phi^{*} L_{k} \cdot \phi^{*} H^{d-k}\right\rangle_{\widetilde{X} \mid \widetilde{V}} \\
& \geq\left\langle\phi^{*} L_{1} \cdots \phi^{*} L_{k} \cdot A^{d-k}\right\rangle_{\widetilde{X} \mid \widetilde{V}} \\
& =\left\langle\phi^{*} L_{1} \cdots \phi^{*} L_{k}\right\rangle_{\widetilde{X} \mid \widetilde{V}} \cdot A^{d-k}>0 .
\end{aligned}
$$

We next consider how the restricted positive product behaves when passing to an admissible model.

Proposition 4.20. Let $X$ be a smooth variety, $V$ a subvariety, and $L_{1}, \ldots, L_{k} V$ pseudoeffective divisors. Suppose $f:(Y, W) \rightarrow(X, V)$ is an admissible model. Then

$$
f_{*}\left\langle f^{*} L_{1} \cdots \cdot f^{*} L_{k}\right\rangle_{Y \mid W}=\operatorname{deg}\left(\left.f\right|_{W}\right)\left\langle L_{1} \cdots L_{k}\right\rangle_{X \mid V} .
$$

Note that $f^{*} L_{i}$ is $W$-pseudoeffective by Proposition 2.5 .

Proof. It suffices to consider the case when the $L_{i}$ are $V$-big. By Lemma 2.8, we may suppose that $L_{i} \geq_{V} 0$. Let $\phi_{m}: X_{m} \rightarrow X$ be a sequence of $V$-birational models that computes $\left\langle L_{1} \cdots L_{k}\right\rangle_{X \mid V}$, and let $\psi_{m}: Y_{m} \rightarrow Y$ be a sequence of $W$-birational models that computes $\left\langle f^{*} L_{1} \cdots \cdots f^{*} L_{k}\right\rangle_{Y \mid W}$. Since the natural map $\phi_{m}^{-1} \circ f \circ \psi_{m}$ is a morphism on the generic point of $W$, by passing to higher $W$-birational models, we may assume that $Y_{m}$ admits a morphism $f_{m}: Y_{m} \rightarrow X_{m}$. Note that

$$
f_{m}^{*} N_{i, m} \leq \widetilde{W}_{m} P_{\sigma}\left(\psi_{m}^{*} f_{m}^{*} L_{i}\right) \leq \widetilde{W}_{m} f_{m}^{*} P_{\sigma}\left(\phi_{m}^{*} L_{i}\right) \leq_{\widetilde{W}_{m}} f_{m}^{*} N_{i, m}+\frac{1}{m} f_{m}^{*} \phi_{m}^{*} G_{i} .
$$

By construction, the pushforwards

$$
\phi_{m *} f_{m *}\left(f_{m}^{*} N_{1, m} \cdots \cdot f_{m}^{*} N_{k, m} \cdot \widetilde{W}_{m}\right)
$$

converge to $\operatorname{deg}\left(\left.f\right|_{W}\right)\left\langle L_{1} \cdots \cdots L_{k}\right\rangle_{X \mid V}$. The same is true for the terms on the right-hand side. Thus, $f_{*} \psi_{m *}\left\langle P_{\sigma}\left(\psi_{m}^{*} f^{*} L_{1}\right) \cdots P_{\sigma}\left(\psi_{m}^{*} f^{*} L_{k}\right)\right\rangle_{Y \mid \widetilde{W}_{m}}$ converges to the same thing, and Theorem 4.15 finishes the proof.

It is worth pointing out that Proposition 4.20 does not contradict the invariance of $\operatorname{vol}_{X \mid V}(L)$ under passing to admissible models. Even if $L$ is $V$-big, $\phi^{*} L$ will not be $W$-big when $\operatorname{deg}\left(\left.f\right|_{W}\right)>1$, so Proposition 4.12 does not apply to $W$.

Proposition 4.21. Let $X$ be a smooth variety, $V$ a subvariety of dimension $d$, and $L$ a V-pseudoeffective divisor. Suppose that $\operatorname{deg}\left(\left\langle L^{d}\right\rangle_{X \mid V}\right)>0$. Then for a very general intersection of very ample divisors $W$ of dimension $d$, we also have $\operatorname{deg}\left(\left\langle L^{d}\right\rangle_{X \mid W}\right)>0$. 
Proof. Fix a sequence of maps $\phi_{m}: \widetilde{X}_{m} \rightarrow X$ for $L$ as in Corollary 4.16. By choosing very ample divisors $H_{1}, \ldots, H_{n-d}$ very general in their linear systems, we may ensure that no $H_{i}$ contains any $\phi_{m}$-exceptional center and the intersection $W=H_{1} \cap \cdots \cap H_{n-d}$ is smooth of the expected dimension.

For each $i=1,2, \ldots, n-d$, choose a positive integer $c_{i}$ so that $\Phi_{V}\left(c_{i} H_{i}\right)$ is generated by global sections, and set $C=\prod_{i} c_{i}^{-1}$. Note that for any $V$-birational model $\phi:(Y, \widetilde{V}) \rightarrow(X, V)$, there are $D_{i} \in\left|c_{i} \phi^{*} H_{i}\right|$ such that each $D_{i}$ has multiplicity at least 1 along $\widetilde{V}$ and $D_{1} \cap \cdots \cap D_{n-k}$ has dimension $k$. In particular for $\phi_{m}$,

$$
\begin{aligned}
{[\tilde{W}] } & =C\left[\phi_{m}^{*} c_{1} H_{1}\right] \cap\left[\phi_{m}^{*} c_{2} H_{2}\right] \cap \cdots \cap\left[\phi_{m}^{*} c_{n-d} H_{n-d}\right] \\
& \succeq C[\tilde{V}],
\end{aligned}
$$

where $\widetilde{W}$ and $\widetilde{V}$ denote the strict transforms of $W$ and $V$ on $\widetilde{X}_{m}$. In particular, for any numerically effective divisor $N$ on $\widetilde{X}_{m}$, we have $N^{d} \cdot \widetilde{W} \geq N^{d} \cdot \widetilde{V}$, and the conclusion follows.

\section{Nakayama constants}

Suppose that $L$ is an ample divisor and $V$ is a subvariety in $X$. Let $\phi: Y \rightarrow X$ be a smooth resolution of the ideal $\Phi_{V}$, and define the divisor $E$ by the equation $O_{Y}(-E)=\phi^{-1} \mathscr{I}_{V} \cdot \mathscr{O}_{Y}$. The Seshadri constant

$$
\varepsilon(L, V):=\max \left\{\tau \mid \phi^{*} L-\tau E \text { is numerically effective }\right\}
$$

measures "how ample" $L$ is along the subvariety $V$. Seshadri constants play an important role in understanding the positivity properties of ample divisors. We will be interested in a related notion that can be defined for an arbitrary pseudoeffective divisor $L$. It first appears in connection with the numerical dimension in [Nakayama 2004].

Definition 5.1. Let $X$ be a normal variety, $\mathscr{I}$ an ideal sheaf on $X$, and $L$ a pseudoeffective divisor. Choose a smooth resolution $\phi: Y \rightarrow X$ of $\mathscr{I}$, and define $E$ by setting $O_{Y}(-E)=\phi^{-1} \mathscr{I} \cdot \bigcirc_{Y}$. We define the Nakayama constant

$$
\varsigma(L, \mathscr{I}):=\max \left\{\tau \mid \phi^{*} L-\tau E \text { is pseudoeffective }\right\} .
$$

Of course, $\varsigma$ is independent of the choice of resolution. When $\mathscr{I}$ is the ideal sheaf of a subvariety $V, \varsigma(L, V)$ will denote the Nakayama constant.

One advantage of $\varsigma(L, V)$ is that it can be positive even when $L$ is pseudoeffective but not big. Thus, the Nakayama constant is a more sensitive measure of positivity than the moving Seshadri constant of [Nakamaye 2003], which always vanishes as we approach the pseudoeffective boundary. It turns out that the Nakayama constant is closely related to the other notions of positivity we have considered. 
Remark 5.2. Nakayama [2004] works with a slightly different formulation of this concept. His definition is equivalent to ours; the equivalence is demonstrated in the first paragraph of the proof of Proposition 5.3.

There is a useful criterion for nonvanishing of $\varsigma$ that is closer in spirit to Nakayama's original formulation.

Proposition 5.3. Let $X$ be a normal variety, $\mathscr{I}$ an ideal sheaf, and $L$ a pseudoeffective divisor. Then $\varsigma(L, \mathscr{I})>0$ if and only if there is an ample divisor $A$ on $X$ so that for any $q$

$$
h^{0}\left(X, \overline{\Phi^{q}} \otimes \mathcal{O}_{X}(\lceil m L\rceil+A)\right)>0
$$

for sufficiently large $m$, where $\overline{\Phi^{q}}$ denotes the integral closure of $\Phi^{q}$.

Note that we can replace $\lceil-\rceil$ by $\lfloor-\rfloor$ by absorbing the difference into $A$.

Proof. Let $\phi: Y \rightarrow X$ denote a smooth resolution of $\mathscr{I}$ and $\mathscr{O}_{Y}(-E)=\phi^{-1} \mathscr{g} \cdot \mathscr{O}_{Y}$ define $E$. Suppose that $\varsigma(L, \mathscr{T})=0$ so that $m \phi^{*} L-E$ is not pseudoeffective for any $m$. Let $p: N^{1}(Y) \rightarrow V$ denote the cokernel of the inclusion $\mathbb{R}\left[\phi^{*} L\right] \rightarrow N^{1}(Y)$. Note that $p(-E)$ is disjoint from $p\left(\overline{N E}^{1}(Y)\right)$. Thus, there is a small ample divisor $H$ on $Y$ so that $p(-E+H)$ is still disjoint from $p\left(\overline{N E}^{1}(Y)\right)$. In other words, $m \phi^{*} L-E+H$ is not pseudoeffective for any $m$.

Let $A$ be any ample divisor on $X$. Choose $q$ so that $q H-\phi^{*} A$ is pseudoeffective. Then $m \phi^{*} L-q E+\phi^{*} A$ is not pseudoeffective for any $m$. Thus, for any $A$ there is a $q$ so that

$$
h^{0}\left(Y, \mathscr{O}_{Y}\left(\phi^{*}(\lfloor m L\rfloor+A)-q E\right)\right)=0
$$

for every $m$. Since the class of $\lceil m L\rceil-\lfloor m L\rfloor$ is bounded as $m$ varies, by absorbing the difference into $A$, the condition using $\lceil m L\rceil$ also fails.

Conversely, suppose that $\varsigma(L, \mathscr{I})>0$. Then for any real number $b>0$, $a \phi^{*} L-b E$ is pseudoeffective for any $a \geq b / \varsigma(L, \mathscr{I})$. By Proposition 2.17 (and Remark 2.15), there is an ample divisor $H$ on $Y$ (independent of $b$ ) so that

$$
h^{0}\left(Y, \mathcal{O}_{Y}\left(\left\lfloor c\left(a \phi^{*} L-b E\right)\right\rfloor+H\right)\right)>0
$$

for every $c>0$ and every $a \geq b / \varsigma(L, \mathscr{I})$. Choose an ample $\mathbb{Z}$-divisor $A \geq \phi_{*} H$. Then $\phi^{*} A \geq \phi^{*} \phi_{*} H \geq H$ so that

$$
h^{0}\left(Y, \mathscr{O}_{Y}\left(\phi^{*}(\lceil a c L\rceil+A)-\lfloor b c E\rfloor\right)\right)>0 .
$$

Fix an integer $q$ and choose $c$ so that $\lfloor c b E\rfloor \geq q E$. Then for any $m>b c / \varsigma(L, \mathscr{g})$,

$$
h^{0}\left(X, \overline{\Phi^{q}} \otimes \mathcal{O}_{X}(\lceil m L\rceil+A)\right)>0 .
$$

If we are only interested in whether $\varsigma(L, \mathscr{I})>0$, we can replace the condition of Proposition 5.3 by several alternatives. We have $\Phi^{q} \subset \overline{\Phi q} \subset g^{\langle q\rangle}$, and by the comparison theorems for symbolic powers (for example, [Swanson 2000, Theorem 3.1]), 
there is some $k$ independent of $q$ so that $\Phi^{\langle k q\rangle} \subset \Phi^{q}$. When $X$ is smooth, we have $\Im^{q} \subset \mathscr{S}^{\left(\Im^{q}\right)}$, and by Skoda's theorem, $\mathscr{g}\left(\Phi^{q}\right) \subset \Phi^{q-\operatorname{dim} X+1}$ for sufficiently large $q$. Thus, the nonvanishing of $\varsigma(L, \mathscr{I})$ is equivalent to the statement that for any $q$

$$
h^{0}\left(X, *_{q} \otimes \mathcal{O}_{X}(\lceil m L\rceil+A)\right)>0
$$

for sufficiently large $m$, where $*_{q}$ can be

- $g^{q}$

- $\Phi^{\langle q\rangle}$, or

- $\mathscr{g}\left(g^{q}\right)$ when $X$ is smooth.

Applying the statement for symbolic powers, we immediately get the following:

Proposition 5.4. Let $X$ be a normal variety, $V$ a subvariety not contained in $\operatorname{Sing}(X)$, and $L$ a divisor. If $(\tilde{X}, \widetilde{V})$ is a smooth $V$-birational model for $(X, V)$, then $\varsigma\left(\phi^{*} L, \widetilde{V}\right)>0$ if and only if $\varsigma(L, V)>0$.

The following proposition indicates that the Nakayama constant satisfies the usual compatibility relations:

Proposition 5.5. Let $X$ be a smooth variety, let $L$ be a pseudoeffective divisor, and let $\Phi$ be an ideal such that no associated prime of $\Phi$ is centered in $\mathbf{B}_{-}(L)$. Then

(1) $\varsigma(L, \mathscr{I})=\varsigma\left(P_{\sigma}(L), \mathscr{P}\right)$, and

(2) if $L$ is big, then $\varsigma(L, \mathscr{I})=\max _{\phi^{*} L \geq A} \varsigma\left(A, \phi^{-1} \mathscr{I} \cdot O_{Y}\right)$, where $\phi: Y \rightarrow X$ varies over all birational maps and $A$ is big and numerically effective.

Proof. (1) It suffices to show the inequality $\leq$. Let $\phi: Y \rightarrow X$ denote a smooth resolution of $\mathscr{I}$, and let $E$ denote the divisor satisfying $\mathscr{O}_{X}(-E)=\phi^{-1} \mathscr{I} \cdot \mathscr{O}_{Y}$. Suppose that $\phi^{*} L-\tau E$ is pseudoeffective. Fix an ample $A$ on $Y$. For any $\epsilon>0$, we find that $\phi^{*} L+\epsilon A \sim_{\mathbb{R}} \tau E+F$ for some effective $F$. Since $\operatorname{Supp}(E)$ is not contained in the diminished base locus of $\phi^{*} L$, we know that $N_{\sigma}\left(\phi^{*} L+\epsilon A\right) \leq F$. Subtracting, we find that $P_{\sigma}\left(\phi^{*} L+\epsilon A\right)-\tau E$ is pseudoeffective. Taking a limit over $\epsilon$ and noting that $\phi^{*} P_{\sigma}(L) \geq P_{\sigma}\left(\phi^{*} L\right)$ completes the proof of the inequality.

(2) It suffices to show the inequality $\leq$. We may also replace $L$ by some $\mathbb{Q}$ linearly equivalent divisor so that $L \geq 0$. Fix an effective ample divisor $H$ on $X$. Proposition 3.7 indicates that there are birational maps $\phi_{m}$ and big and numerically effective divisors $N_{m}$ satisfying $N_{m} \leq P_{\sigma}\left(\phi_{m}^{*} L\right) \leq N_{m}+\frac{1}{m} \phi_{m}^{*} H$. The expression on the right-hand side can be made arbitrarily close to $\varsigma\left(P_{\sigma}(L), \phi^{-1} \mathscr{g} \cdot \mathscr{O}_{Y}\right)$. By (1), this equals $\varsigma(L, \mathscr{I})$.

[Nakayama 2004] shows that $\varsigma(L, V)$ is controlled by what happens to a very general subvariety of dimension equal to $\operatorname{dim} V$. 
Proposition 5.6 [Nakayama 2004, Lemma V.2.21]. Let $X$ be a smooth variety of dimension $n$, and let $L$ be a pseudoeffective divisor. Suppose there is a $d$ dimensional subvariety $V$ such that $\varsigma(L, V)=0$. Then there is a very ample divisor $H$ so that any complete intersection $W$ of $(n-d)$ very general elements of $|H|$ satisfies $\varsigma(L, W)=0$.

\section{The numerical dimension}

Our goal in this section is to show that the different definitions of the numerical dimension coincide. We start by giving an example of effective divisors that are numerically equivalent but have different litaka dimensions.

Example 6.1. We give an example of a threefold $X$ and effective divisors $L$ and $L^{\prime}$ so that $L \equiv L^{\prime}$ but $\kappa(L) \neq \kappa\left(L^{\prime}\right)$. Fix an elliptic curve $E$, and consider $S=E \times E$ with projection maps $p_{1}$ and $p_{2}$. Let $F$ be a fiber of $p_{1}$. Choose a degree- 0 divisor $T$ on $E$ that is nontorsion, and define $N=p_{2}^{*} T$. We have $\kappa(F)=1$ and $\kappa(F+N)=-\infty$.

Let $X$ be the $\mathbb{P}^{1}$-bundle $\mathbb{P}_{S}\left(\mathcal{O}_{S} \oplus \mathcal{O}_{S}(F+N)\right)$ with the morphism $\pi: X \rightarrow S$. Define $L$ to be the section $\mathbb{P}_{S}\left(\mathscr{O}_{S}\right)$, and define $L^{\prime}=L-\pi^{*} N$. Note that $L$ and $L^{\prime}$ are numerically equivalent. By identifying the pushforwards of $O_{X}(m L)$ with symmetric powers of $\mathrm{O}_{S} \oplus \mathrm{O}_{S}(F+N)$, we see that $\kappa(L)=0$. Similarly, since $\mathcal{O}_{X}\left(L^{\prime}\right)$ can be realized as the relative dualizing sheaf of $\mathbb{P}_{S}\left(\mathscr{O}_{S}(-N) \oplus \mathscr{O}_{S}(F)\right)$, we see that $\kappa\left(L^{\prime}\right) \geq \kappa(F)=1$.

We first prove Theorem 1.1 for smooth varieties $X$. For convenience, we arrange the definitions in a more suitable order. Definition (1) in the following theorem is the definition of numerical dimension in [Boucksom et al. 2012] while (5) and (6) correspond to $\kappa_{\sigma}(L)$ and $\kappa_{v}(L)$ (by Remark 5.2) in [Nakayama 2004]. Note that we allow varieties $W \subset \operatorname{Supp}(L)$ at the slight cost of using numerical restrictions in (4).

Theorem 6.2. Let $X$ be a smooth variety, and let $L$ be a pseudoeffective divisor. Here $A$ will denote some fixed sufficiently ample $\mathbb{Z}$-divisor and $W$ will range over all subvarieties of $X$ not contained in $\mathbf{B}_{-}(L)$. Then the following quantities coincide:

(1) $\max \left\{k \in \mathbb{Z}_{\geq 0} \mid\left\langle L^{k}\right\rangle_{X} \neq 0\right\}$.

(2) $\max \left\{\operatorname{dim} W \mid\left\langle L^{\operatorname{dim} W}\right\rangle_{X \mid W}>0\right\}$.

(3) $\max \left\{\operatorname{dim} W \mid \lim _{\epsilon \rightarrow 0} \operatorname{vol}_{X \mid W}(L+\epsilon A)>0\right\}$.

(4) $\max \left\{\operatorname{dim} W \mid \inf _{\phi} \operatorname{vol}_{\widetilde{W}}\left(\left.\left[P_{\sigma}\left(\phi^{*} L\right)\right]\right|_{\widetilde{W}}\right)>0\right\}$, where $\phi:(\tilde{X}, \widetilde{W}) \rightarrow(X, W)$ ranges over $W$-birational models.

(5) $\max \left\{k \in \mathbb{Z}_{\geq 0} \mid \lim \sup _{m \rightarrow \infty} h^{0}(X,\lfloor m L\rfloor+A) / m^{k}>0\right\}$.

(6) $\min \{\operatorname{dim} W \mid \varsigma(L, W)=0\}$ (by convention, if $L$ is big we interpret this expression as returning $\operatorname{dim} X)$.

(7) $\max \left\{k \in \mathbb{Z}_{\geq 0} \mid \exists C>0\right.$ such that $C t^{n-k}<\operatorname{vol}(L+t A)$ for all $\left.t>0\right\}$. 
We call this common quantity the numerical dimension of $L$ and denote it $v_{X}(L)$. It only depends on the numerical class of $L$.

We will prove Theorem 6.2 using a cycle of inequalities. The equivalence of (1)-(4) is an easy consequence of the properties of the positive product, and the inequality $(5) \leq$ (6) was proved in [Nakayama 2004, Proposition V.2.22]. The other inequalities will require more work.

Proof. $(1)=(2)$. Let $H_{1}, \ldots, H_{d-k}$ represent very general elements of a very ample linear system. Since $\left\langle L^{k}\right\rangle_{X}$ is in the closure of the cone generated by effective cycles, it is nonzero if and only if $\left\langle L^{k}\right\rangle_{X} \cdot H_{1} \cdots \cdot H_{d-k}>0$. By Lemma 4.18, this is equivalent to $\left\langle L^{k}\right\rangle_{X \mid H_{1} \cap \cdots \cap H_{d-k}}>0$. Thus, $(1) \leq(2)$. By Proposition 4.21, the same argument in reverse shows that $(2) \leq(1)$.

$(2)=(3)$. Proposition 4.12 shows that the conditions set on $W$ in (2) and (3) are the same.

$(3)=(4)$. Proposition 4.12 allows us to translate between restricted volume and the restricted positive product in the $V$-big case. Thus, Theorem 4.15 implies that

$$
\operatorname{vol}_{X \mid W}(L+\epsilon A)=\inf _{\phi: \widetilde{X} \rightarrow X} \operatorname{vol}_{\widetilde{W}}\left(\left.\left[P_{\sigma}\left(\phi^{*}(L+\epsilon A)\right)\right]\right|_{\widetilde{W}}\right),
$$

where $\phi:(\widetilde{X}, \widetilde{W}) \rightarrow(X, W)$ varies over $W$-birational models. Consider

$$
\lim _{\epsilon \rightarrow 0} \operatorname{vol}_{X \mid W}(L+\epsilon A)=\lim _{\epsilon \rightarrow 0} \inf _{\phi: \widetilde{X} \rightarrow X} \operatorname{vol}_{\widetilde{W}}\left(\left.\left[P_{\sigma}\left(\phi^{*}(L+\epsilon A)\right)\right]\right|_{\widetilde{W}}\right) .
$$

Note that on any model $\operatorname{vol}_{\widetilde{W}}\left(\left.\left[P_{\sigma}\left(\phi^{*}(L+\epsilon A)\right)\right]\right|_{\widetilde{W}}\right)$ is nondecreasing and continuous as a function of $\epsilon$. Thus, on the right-hand side, we may commute the limit with the infimum.

(4) $\leq(5)$. The first step is to show that there is some ample divisor on $W$ whose pullback lies beneath each restriction $\left.P_{\sigma}\left(\phi^{*} L\right)\right|_{\widetilde{W}}$. Using this ample divisor, we find a lower bound for the growth of sections of a certain twisted linear series on $W$. The last step is to prove a lifting theorem for twisted linear series to conclude that $h^{0}(\lfloor m L\rfloor+A)$ satisfies the necessary growth conditions.

Lemma 6.3. Let $X$ be a smooth variety of dimension $n$, let $L$ be a big divisor, and let $N$ be a general element of a big basepoint-free linear system. Then we have $\operatorname{vol}(L-N) \geq \operatorname{vol}(L)-n \operatorname{vol}_{X \mid N}(L)$.

The easiest demonstration appeals to the results of [Boucksom et al. 2009].

Proof. Let $\alpha=\sup _{t \in[0,1]}\{L-t N$ is pseudoeffective $\}$. Note $1 \geq \alpha$, and since $L$ is big, $0<\alpha$. We will prove the stronger result $\operatorname{vol}(L-N) \geq \operatorname{vol}(L)-n \alpha \operatorname{vol}_{X \mid N}(L)$. 
By [Boucksom et al. 2009, Corollary C], the function vol is continuously differentiable on the big cone. More precisely, for $t \in(0, \alpha)$ we have

$$
\frac{d}{d t} \operatorname{vol}(L-t N)=-n \operatorname{vol}_{X \mid N}(L-t N) .
$$

Note that $\operatorname{vol}_{X \mid N}(L-t N) \leq \operatorname{vol}_{X \mid N}(L)$ for any $t \geq 0$. Thus, for every $t \in(0, \alpha)$ there is an inequality

$$
\frac{d}{d t} \operatorname{vol}(L-t N) \geq-n \operatorname{vol}_{X \mid N}(L) .
$$

Integrating both sides over $t \in[0, \alpha]$, we get $\operatorname{vol}(L-\alpha N) \geq \operatorname{vol}(L)-n \alpha \operatorname{vol}_{X \mid N}(L)$. But if $\alpha \neq 1$, then $\operatorname{vol}(L-\alpha N)=0=\operatorname{vol}(L-N)$, finishing the proof.

Lemma 6.4. Let $W$ be a smooth variety. Suppose that for every smooth birational model $\phi: \widetilde{W} \rightarrow W$ we associate a divisor $L_{\widetilde{W}}$ so that for any birational map $\psi: \widehat{W} \rightarrow \widetilde{W}$ we have $\psi^{*} L_{\widetilde{W}} \geq L_{\widehat{W}}$. Suppose furthermore that

$$
\inf _{\widetilde{W}} \operatorname{vol}\left(L_{\widetilde{W}}\right)>0 .
$$

There is some ample divisor $H$ on $W$ and constant $\epsilon$ such that $\operatorname{vol}\left(L_{\widetilde{W}}-\phi^{*} H\right)>\epsilon$ for every $\phi$.

Note that $\operatorname{vol}\left(L_{\widetilde{W}}\right) \geq \operatorname{vol}\left(L_{\widehat{W}}\right)$ for every higher model $\widehat{W}$.

Proof. For convenience, set $n=\operatorname{dim} W$ and $\tau=\inf \operatorname{vol}\left(L_{\widetilde{W}}\right)$. Fix a very ample divisor $H$ on $W$. It suffices to show that there is some constant $k$ such that for any smooth model $\phi: \widetilde{W} \rightarrow W$, there is an $H^{\prime} \equiv H$ so that

$$
\operatorname{vol}\left(L_{\widetilde{W}}-\frac{1}{k} \phi^{*} H^{\prime}\right)>\tau / 2 .
$$

Choose a prime very ample divisor $H^{\prime} \equiv H$ sufficiently general so that $\psi^{*} H^{\prime}$ is equal to the strict transform of $H^{\prime}$. Note that

$$
\operatorname{vol}_{\widetilde{W} \mid \phi^{*} H^{\prime}}\left(L_{\widetilde{W}}\right) \leq \operatorname{vol}_{\widetilde{W} \mid \phi^{*} H^{\prime}}\left(\phi^{*} L_{W}\right),
$$

and by [Ein et al. 2009, Lemma 2.4], the latter quantity is equal to $\operatorname{vol}_{W \mid H^{\prime}}\left(L_{W}\right)$. Choose some constant $k$ so that

$$
\frac{1}{k} \operatorname{vol}_{W \mid H^{\prime}}\left(L_{W}\right)<\frac{\tau}{2 n} .
$$

(Note that by [Boucksom et al. 2009, Proposition 4.8], $k$ is independent of the choice of $H^{\prime}$ and thus also independent of the choice of $\widetilde{W}$.) Lemma 6.3 implies

$$
\begin{aligned}
\operatorname{vol}\left(k L_{\widetilde{W}}-\phi^{*} H^{\prime}\right) & \geq \operatorname{vol}\left(k L_{\widetilde{W}}\right)-n \operatorname{vol}_{\widetilde{W} \mid \phi^{*} H^{\prime}}\left(k L_{\widetilde{W}}\right) \\
& \geq \operatorname{vol}\left(k L_{\widetilde{W}}\right)-n \operatorname{vol}_{\widetilde{W} \mid \phi^{*} H^{\prime}}\left(k \phi^{*} L_{W}\right) .
\end{aligned}
$$


Rescaling the above expression by $k$, we find

$$
\operatorname{vol}\left(L_{\widetilde{W}}-\frac{1}{k} \phi^{*} H^{\prime}\right) \geq \operatorname{vol}\left(L_{\widetilde{W}}\right)-\frac{n}{k} \operatorname{vol}_{\widetilde{W} \mid \phi^{*} H^{\prime}}\left(\phi^{*} L_{W}\right)>\tau / 2 .
$$

In our situation, we find the following:

Corollary 6.5. Assume that $W$ is a very general intersection of very ample divisors such that $\inf _{\phi} \operatorname{vol}_{\widetilde{W}}\left(\left.P_{\sigma}\left(\phi^{*} L\right)\right|_{\widetilde{W}}\right)>0$, where $\phi:(\tilde{X}, \widetilde{W}) \rightarrow(X, W)$ varies over all $W$-birational models. Then there is an ample divisor $H$ on $W$ so that for any $W$-birational model $\phi: \widetilde{X} \rightarrow X$, we have

$$
\operatorname{vol}_{\widetilde{W}}\left(\left.P_{\sigma}\left(\phi^{*} L\right)\right|_{\widetilde{W}}-\phi^{*} H\right)>0 .
$$

Proof. Consider the set of divisors $\left.P_{\sigma}\left(\phi^{*} L\right)\right|_{\widetilde{W}}$. Since $N_{\sigma}\left(\phi^{*} L\right) \geq \widetilde{W} 0$, they satisfy the comparison condition of Lemma 6.4. By assumption, the infimum condition of Lemma 6.4 also holds. The lemma yields an appropriate ample divisor $H$ on $W$.

Our next goal is a lifting theorem for twisted linear series.

Proposition 6.6. Let $X$ be a smooth variety, and let $L$ be an effective divisor. Suppose that $N$ is a big and numerically effective divisor satisfying $0 \leq N \leq L$ such that $N$ has simple normal crossing support. Let $|B|$ be a basepoint-free linear system defining a birational morphism on X. For sufficiently general elements $B_{1}, \ldots, B_{k} \in|B|$, we have an inequality

$h^{0}\left(W, \mathscr{O}_{W}\left(K_{W}+\left\lceil\left. N\right|_{W}\right\rceil+\left.A\right|_{W}\right)\right) \leq h^{0}\left(X \mid W, \mathscr{O}_{X}\left(K_{X}+\lceil L\rceil+B_{1}+\cdots+B_{k}+A\right)\right)$,

where $W$ is the complete intersection $B_{1} \cap \cdots \cap B_{k}$ and $A$ is any numerically effective $\mathbb{Z}$-divisor on $X$.

Proof. For convenience, define $W_{j}:=B_{1} \cap \cdots \cap B_{j}$ and $M_{i}:=B_{i+1}+\cdots+B_{k}$. Note that since the $B_{i}$ are sufficiently general, we may assume that each $W_{j}$ is smooth, that $N \geq_{W_{j}} 0$, and that $\left.N\right|_{W_{j}}$ has simple normal crossing support. Note furthermore that $B$ is big and numerically effective so that $\left.M_{i}\right|_{W_{j}}$ is also a big and numerically effective divisor for any $i$ and $j$.

Kawamata-Viehweg vanishing implies that we have surjections

$$
\begin{aligned}
H^{0}\left(W_{i}, \mathcal{O}_{W_{i}}\left(K_{W_{i}}+\left\lceil\left. N\right|_{W_{i}}\right\rceil+\left.\left(A+M_{i}\right)\right|_{W_{i}}\right)\right) \rightarrow \\
H^{0}\left(W_{i+1}, \mathcal{O}_{W_{i+1}}\left(K_{W_{i+1}}+\left.\left\lceil\left. N\right|_{W_{i}}\right\rceil\right|_{W_{i+1}}+\left.\left(A+M_{i+1}\right)\right|_{W_{i+1}}\right)\right) .
\end{aligned}
$$

Furthermore, since $N \geq{ }_{W_{i}} 0$ for every $i$, we have $\left.\left\lceil\left. N\right|_{W_{i}}\right\rceil\right|_{W_{i+1}} \geq\left\lceil\left. N\right|_{W_{i+1}}\right\rceil$. Thus, by induction we obtain

$$
\begin{aligned}
h^{0}\left(X \mid W_{i}, \mathrm{O}_{X}(\lceil N\rceil+(\right. & \left.\left.\left.K_{X}+A+B_{1}+\cdots+B_{k}\right)\right)\right) \\
& \geq h^{0}\left(W_{i}, O_{W_{i}}\left(\left\lceil\left. N\right|_{W_{i}}\right\rceil+\left.\left(K_{X}+A+B_{1}+\cdots+B_{k}\right)\right|_{W_{i}}\right)\right) .
\end{aligned}
$$

When $i=k$, we obtain the desired statement. 
We now finish the proof of the inequality (4) $\leq$ (5). Set $k$ to be the value of (4). Fix an ample divisor $A$ on $X$ as in Theorem 2.4 so that for any $m$ there is an $L_{m} \sim\lceil m L\rceil+A$ such that $L_{m} \geq 0$.

For each $L_{m}$, we can apply Proposition 3.7 to find an effective divisor $G_{m}$, a countable sequence of maps $\phi_{i, m}$, and a big and numerically effective divisor $N_{i, m}$ satisfying

$$
N_{i, m} \leq P_{\sigma}\left(\phi_{i, m}^{*} L_{m}\right) \leq N_{i, m}+\frac{1}{i} \phi_{i, m}^{*} G_{m} .
$$

We may of course assume that each $N_{i, m}$ has simple normal crossing support and each $\phi_{i, n}$ is a composition of blowups along smooth centers.

Note that the set of maps $\phi_{i, m}$ is countable as $m$ and $i$ vary. Fix a very ample linear system $|B|$ on $X$. We can choose very general elements $B_{1}, \ldots, B_{k} \in|B|$ so that the $\phi_{i, m}^{*} B_{j}$ satisfy the conditions of Proposition 6.6 for each $\widetilde{X}_{i, m}$ and $N_{i, m}$ simultaneously. We may also choose the $B_{j}$ sufficiently general so that the strict transform of $B_{j}$ over $\phi_{i, m}$ is the same as the pullback for every $i$ and $m$. Set $W=B_{1} \cap \cdots \cap B_{k}$. Then each $\phi_{i, m}$ is $W$-birational and $\widetilde{W}_{i, m, j}=\phi_{i, m}^{*} B_{1} \cap \cdots \cap \phi_{i, m}^{*} B_{j}$ is smooth for every $j$ between 1 and $k$.

Choose an ample divisor $H$ on $W$ as in Corollary 6.5. For each $G_{m}$, choose a sufficiently small $\epsilon_{m}>0$ so that $H-\left.\epsilon_{m} G_{m}\right|_{W}$ is pseudoeffective. By choosing $i>1 / \epsilon_{m}$, we find models $\phi_{m}: \widetilde{X}_{m} \rightarrow X$ so that

$$
N_{m} \leq \widetilde{W}_{m} P_{\sigma}\left(\phi_{m}^{*} L_{m}\right) \leq \widetilde{W}_{m} N_{m}+\epsilon_{m} \phi_{m}^{*} G_{m} .
$$

Thus,

$$
\begin{aligned}
\left.N_{m}\right|_{\widetilde{W}_{m}}-(m-1) \phi_{m}^{*} H \geq & \left.\left(P_{\sigma}\left(\phi_{m}^{*} L_{m}\right)-\epsilon_{m} \phi_{m}^{*} G_{m}\right)\right|_{\widetilde{W}_{m}}-(m-1) \phi_{m}^{*} H \\
\geq & \left.\left(P_{\sigma}\left(\phi_{m}^{*} L_{m}\right)-P_{\sigma}\left(\phi_{m}^{*} m L\right)\right)\right|_{\widetilde{W}_{m}} \\
& \quad+m\left(\left.P_{\sigma}\left(\phi_{m}^{*} L\right)\right|_{\widetilde{W}_{m}}-\phi_{m}^{*} H\right)+\phi_{m}^{*}\left(H-\left.\epsilon_{m} G_{m}\right|_{W}\right) .
\end{aligned}
$$

We analyze this last sum term by term. Since $L_{m}-m L$ is $W$-pseudoeffective and $N_{\sigma}\left(\phi_{m}^{*} L\right) \geq \widetilde{W}_{m} 0$, the first term is pseudoeffective by Proposition 3.5. The conclusion of Corollary 6.5 is that the second term is big. The third term is also pseudoeffective by construction. Thus, $D_{m}:=\left.N_{m}\right|_{\widetilde{W}_{m}}-(m-1) \phi_{m}^{*} H$ is big.

Fix a very ample divisor $M$ on $X$. Then

$$
\begin{aligned}
h^{0}\left(\widetilde{W_{m}}, \widetilde{O}_{\widetilde{W_{m}}}(\right. & \left.\left.K_{\widetilde{W_{m}}}+\left.(k+2) \phi_{m}^{*} M\right|_{W}+\left\lceil\left. N_{m}\right|_{\widetilde{W}_{m}}\right\rceil\right)\right) \\
& \geq h^{0}\left(\widetilde{W_{m}}, \mathcal{O}_{\widetilde{W_{m}}}\left(K_{\widetilde{W_{m}}}+\left.(k+2) \phi_{m}^{*} M\right|_{W}+\left\lceil D_{m}\right\rceil+\left\lfloor(m-1) \phi_{m}^{*} H\right\rfloor\right)\right) \\
& \geq h^{0}(W,\lfloor(m-1) H\rfloor) \quad \text { by Proposition } 2.17 \\
& \geq C m^{k}
\end{aligned}
$$

for some constant $C>0$ and for $m$ sufficiently large. 
We conclude by applying Proposition 6.6. We have already chosen the divisors $B_{1}, B_{2}, \ldots, B_{k}$ sufficiently general so that their pullbacks satisfy the conditions of the theorem. For convenience, define $A^{\prime}=B_{1}+\cdots+B_{k}$. Proposition 6.6 shows that the dimensions of the spaces of restricted sections

$$
h^{0}\left(\widetilde{X}_{m} \mid \widetilde{W}_{m}, \mathcal{O}_{\widetilde{X}_{m}}\left(K_{\widetilde{X}_{m}}+\phi_{m}^{*}\left(L_{m}+A^{\prime}+(k+2) \phi^{*} M\right)\right)\right)>C m^{k}
$$

for some constant $C>0$ and for sufficiently large $m$. Since $K_{\widetilde{X}_{m} / X}$ is $\phi_{m}$-exceptional, these dimensions are equal to

$$
\begin{aligned}
h^{0}\left(X \mid W, \mathcal{O}_{X}\left(K_{X}+L_{m}+\right.\right. & \left.\left.A^{\prime}+(k+2) \phi^{*} M\right)\right) \\
& =h^{0}\left(X \mid W, O_{X}\left(K_{X}+\lceil m L\rceil+A+A^{\prime}+(k+2) \phi^{*} M\right)\right) .
\end{aligned}
$$

Thus, $h^{0}\left(X, \mathscr{O}_{X}\left(K_{X}+\lceil m L\rceil+A+A^{\prime}+(k+2) \phi^{*} M\right)\right)$ is also bounded below by $C m^{k}$ for sufficiently large $m$.

(5) $\leq$ (6). This is proved in [Nakayama 2004, Proposition V.2.22].

(6) $\leq$ (1). By Proposition 5.6, we may assume that $W$ is a very general intersection of very ample divisors. We need to consider the 0 -case separately. Note that (1) is 0 precisely when $P_{\sigma}(L)$ is numerically trivial. This means that (6) is also 0 . Thus, we can prove that $(6) \leq(1)$ by considering the case where (6) is at least 2 and (1) is at least 1.

Suppose for a contradiction that (1) is less than the value of (6). For convenience, we set $k$ to be the value of (1). Let $W$ be a $k$-dimensional intersection of very general, very ample divisors. Set $\tau=\varsigma(L, W)>0$, and let $\phi: Y \rightarrow X$ be the blowup of $W$ with exceptional divisor $E$.

Fix a very ample divisor $H$ on $Y$. We first analyze $\phi^{*} L+\epsilon H$. Choose models $\psi_{i}: \widetilde{Y}_{i} \rightarrow Y$ computing positive products $\left\langle\left(\phi^{*} L+\epsilon H\right)^{k}\right\rangle_{Y \mid E}$ and $\left\langle\left(\phi^{*} L+\epsilon H\right)^{k+1}\right\rangle_{Y}$. Choose big and numerically effective divisors $A_{i} \leq \psi_{i}^{*}\left(\phi^{*} L+\epsilon H\right)$ on $\widetilde{Y}_{i}$ that compute the product. By Proposition 5.5, $P_{\sigma}\left(\psi_{i}^{*}\left(\phi^{*} L+\epsilon H\right)\right)-\tau \psi_{i}^{*} E$ is always pseudoeffective, so by choosing $\psi_{i}$ appropriately, we may also assume $A_{i}-\frac{\tau}{2} \psi_{i}^{*} E$ is pseudoeffective for each $A_{i}$. Thus, $A_{i}-\frac{\tau}{2} \widetilde{E}$ is also pseudoeffective, where $\widetilde{E}$ denotes the strict transform of $E$ on $\tilde{Y}_{i}$. Then

$$
0 \leq\left(A_{i}-\frac{\tau}{2} \widetilde{E}\right) \cdot A_{i}^{k} \cdot \psi_{i}^{*} H^{d-k-1} .
$$

By taking a limit over pushforwards on all such models, we find

$$
0 \leq\left\langle\left(\phi^{*} L+\epsilon H\right)^{k+1}\right\rangle_{Y} \cdot H^{d-k-1}-\frac{\tau}{2}\left\langle\left(\phi^{*} L+\epsilon H\right)^{k}\right\rangle_{Y \mid E} \cdot H^{d-k-1} .
$$

This is true for all sufficiently small $\epsilon$, so

$$
0 \leq\left\langle\phi^{*} L^{k+1}\right\rangle_{Y} \cdot H^{d-k-1}-\frac{\tau}{2}\left\langle\phi^{*} L^{k}\right\rangle_{Y \mid E} \cdot H^{d-k-1} .
$$


By choosing sufficiently general elements $H_{1}, \ldots, H_{d-k-1} \in|H|$, we may ensure that $E \cap H_{1} \cap \cdots \cap H_{d-k-1}$ maps finitely onto $W$ via $\phi$. Letting the $A_{1}, \ldots, A_{d-k}$ denote the ample divisors whose intersection is $W$, we have

$$
\begin{aligned}
\left\langle\phi^{*} L^{k}\right\rangle_{Y \mid E} \cdot H^{d-k-1} & =\left\langle\phi^{*} L^{k}\right\rangle_{Y \mid E \cap H_{1} \cap \cdots \cap H_{d-k-1}} \\
& =C\left\langle L^{k}\right\rangle_{X \mid W} \\
& =C\left\langle L^{k}\right\rangle_{X} \cdot A_{1} \cdots A_{d-k}
\end{aligned}
$$

for some positive constant $C$. By assumption, this latter quantity is positive, so

$$
0<\left\langle\phi^{*} L^{k+1}\right\rangle_{Y} \cdot H^{d-k-1},
$$

contradicting the fact that $\left\langle L^{k+1}\right\rangle_{X}=0$.

$(7) \leq(1)$. Let $k$ denote the value of (1). Note that

$$
\begin{aligned}
t^{n-k}\left\langle(L+t A)^{k}\right\rangle \cdot A^{n-k} & =\left\langle(L+t A)^{k} \cdot(t A)^{n-k}\right\rangle \\
& \leq\left\langle(L+t A)^{n}\right\rangle .
\end{aligned}
$$

The expression in (1) implies that there is some constant $C$ such that $C<\langle(L+$ $\left.t A)^{k}\right\rangle \cdot A^{n-k}$ for every $t>0$. Thus, we obtain $C t^{n-k}<\operatorname{vol}(L+t A)$ for every $t>0$.

$(1) \leq(7)$. Let $k$ denote the value of (7). For every constant $C$, there is some $t>0$ such that

$$
\left\langle(L+t A)^{n}\right\rangle<C t^{n-k-1} .
$$

This implies that

$$
t^{n-k-1}\left\langle(L+t A)^{k+1}\right\rangle \cdot A^{n-k-1}<C t^{n-k-1}
$$

so that for any $C$ there is some $t$ such that $\left\langle(L+t A)^{k+1}\right\rangle \cdot A^{n-k-1}<C$. Note that the left-hand side is increasing in $t$ so that the inequality must hold for arbitrarily small $t$. Thus, the value of (1) is at most $k$.

The numerical dimension satisfies a number of natural properties. All of the following are checked in [Nakayama 2004, Proposition V.2.7] except for (5) and (7): Theorem 6.7 [Nakayama 2004, Proposition V.2.7]. Let X be a smooth variety, and let $L$ be a pseudoeffective $\mathbb{R}$-divisor.

(1) We have $0 \leq v(L) \leq \operatorname{dim} X$ and $\kappa(L) \leq v(L)$.

(2) We have $v(L)=\operatorname{dim} X$ if and only if $L$ is big and $v(L)=0$ if and only if $P_{\sigma}(L) \equiv 0$.

(3) If $L^{\prime}$ is pseudoeffective, then $v\left(L+L^{\prime}\right) \geq v(L)$.

(4) If $f: Y \rightarrow X$ is any surjective morphism from a normal variety $Y$, then $v\left(f^{*} L\right)=v(L)$. 
(5) We have $v(L)=v\left(P_{\sigma}(L)\right)$.

(6) Suppose that $f: X \rightarrow Z$ has connected fibers and $F$ is a very general fiber of $f$. Then $v(L) \leq v\left(\left.L\right|_{F}\right)+\operatorname{dim} Z$.

(7) Fix some sufficiently ample $\mathbb{Z}$-divisor $A$. Then there are positive constants $C_{1}$ and $C_{2}$ so that

$$
C_{1} m^{\nu(L)}<h^{0}\left(X, O_{X}(\lfloor m L\rfloor+A)\right)<C_{2} m^{\nu(L)}
$$

for every sufficiently large $m$.

Proof. Part (5) follows from the invariance of the positive product under passing to $P_{\sigma}$.

Consider the inequality of (7). The leftmost inequality was stated explicitly while demonstrating the implication $(4) \leq(5)$ in the proof of Theorem 6.2. To show the rightmost inequality, let $W$ be a subvariety of dimension $v(L)$ with $\varsigma(L, W)=0$. Proposition 5.3 (and the following discussion) shows that there is a positive integer $q$ with

$$
h^{0}\left(X, g_{W}^{q} \otimes \bigcirc_{X}(\lceil m L\rceil+A)\right)=0
$$

for sufficiently large $m$. Writing $W_{q}$ for the subscheme defined by the ideal $q_{W}^{q}$, for sufficiently large $m$ there is an injection

$$
h^{0}\left(X, \bigcirc_{X}(\lceil m L\rceil+A)\right) \rightarrow h^{0}\left(W_{q}, \bigcirc_{W_{q}}(\lceil m L\rceil+A)\right),
$$

and the rate of growth of the latter is bounded by $m^{\operatorname{dim}\left(W_{q}\right)}=m^{\nu(L)}$.

It is interesting to note that $v$ is not lower semicontinuous as might be expected. This is a consequence of the fact that the restricted positive product is only semicontinuous on the boundary of the $V$-pseudoeffective cone.

Example 6.8 [Boucksom et al. 2009, Example 3.8]. Let $X$ be any smooth surface with infinitely many -1 -curves. Take some compact slice of $\overline{N E}^{1}(X)$. We can choose a convergent sequence of distinct classes $\left\{\alpha_{i}\right\}$ on this compact slice such that each $\alpha_{i}$ lies on a ray generated by a different -1 -curve. Note that for any irreducible curve $C$, there is at most one $i$ for which $\alpha_{i} \cdot C<0$. Thus, $\beta:=\lim _{i \rightarrow \infty} \alpha_{i}$ must be a numerically effective class. A nontrivial numerically effective class $\beta$ has $\nu(\beta) \geq 1$ but $v\left(\alpha_{i}\right)=0$ for every $i$. Thus, $v$ is not lower semicontinuous.

Question 6.9. What properties does $v$ satisfy along the $V$-pseudoeffective boundary?

6A. The numerical dimension for normal varieties. Since the numerical dimension is a birational invariant, we can extend the definition to any normal variety $X$.

Definition 6.10. Let $X$ be a normal variety, and let $L$ be an $\mathbb{R}$-Cartier divisor on $X$. We define $v(L)$ to be $v\left(f^{*} L\right)$, where $f: Y \rightarrow X$ is any smooth model. 
We now complete the proof of Theorem 1.1 by showing that the criteria of Theorem 6.2 can be applied directly to a normal variety. Note that the numbering in the two theorems is different; we will use the numbering of Theorem 1.1.

Proof of Theorem 1.1. We have $(1)=v(L)$ since the arguments in the proof of [Nakayama 2004, Proposition V.2.7] show that (1) is a birational invariant even for normal varieties.

We next show that $(3)=v(L)$. We first claim there is a complete intersection $W$ of very general, very ample divisors that maximizes (3). Suppose that $V \subset X$ is a $k$ dimensional subvariety that achieves the maximum value in (3). Choose very ample divisors $A_{1}, \ldots, A_{n-k}$ whose (scheme-theoretic) complete intersection $W_{0}$ contains $V$ and also has dimension $k$. Set $P=\mathbb{P} H^{0}\left(X, O_{X}\left(A_{1}\right)\right) \times \cdots \times \mathbb{P} H^{0}\left(X, O_{X}\left(A_{n-k}\right)\right)$.

Let $g$ be the ideal sheaf on $X \times P$ whose restriction to a fiber of the second projection is the ideal sheaf of the corresponding complete intersection on $X$. Note that $\mathscr{F}$ is flat over the locus on $P$ representing intersections of the expected dimension. By upper-semicontinuity, we find that for any fixed divisor $D$ we have

$$
h^{0}\left(X, \Phi_{W}(\lfloor D\rfloor)\right) \leq h^{0}\left(X, \mathscr{I}_{W_{0}}(\lfloor D\rfloor)\right)
$$

for a general complete intersection $W$. Thus,

$$
\begin{aligned}
h^{0}\left(X \mid W, \mathscr{O}_{X}(\lfloor D\rfloor)\right) & \geq h^{0}\left(X \mid W_{0}, \mathscr{O}_{X}(\lfloor D\rfloor)\right) \\
& \geq h^{0}\left(X \mid V, \mathcal{O}_{X}(\lfloor D\rfloor)\right)
\end{aligned}
$$

since the restriction map $\mathrm{O}_{X} \rightarrow \mathrm{O}_{V}$ factors through restriction to $\mathfrak{O}_{W_{0}}$. In particular, if we fix a countable collection of divisors $D_{i}$, then for a very general complete intersection $W$, we have $\operatorname{vol}_{X \mid W}\left(D_{i}\right) \geq \operatorname{vol}_{X \mid V}\left(D_{i}\right)$ for every $i$. Setting $D_{i}:=L+\frac{1}{i} A$ yields the claim.

Let $\phi: Y \rightarrow X$ be a smooth model of $X$. For any ample divisor $A$ on $Y$, there is an ample divisor $H$ on $X$ such that $\phi^{*} H \geq A$. Since $W$ is not contained in any $\phi$-exceptional center, we may furthermore ensure that $\operatorname{Supp}\left(\phi^{*} H-A\right)$ does not contain $W$.

In particular, for any ample divisor $A$ on $Y$ there is some $H$ on $X$ such that

$$
\operatorname{vol}_{X \mid W}(L+\epsilon H)=\operatorname{vol}_{Y \mid \widetilde{W}}\left(\phi^{*}(L+\epsilon H)\right) \geq \operatorname{vol}_{Y \mid \widetilde{W}}\left(\phi^{*} L+\epsilon A\right) .
$$

Similarly, for any ample divisor $H$ on $X$ there is an $A$ on $Y$ with $A-\phi^{*} H$ ample. Thus, (3) $=v(L)$ is proved.

Then $(2)=v(L)$ follows from the arguments of the previous two paragraphs, (4) $=v(L)$ since (4) remains unchanged upon passing to a smooth $V$-birational model, both $(5)=v(L)$ and $(6)=v(L)$ follow from Corollary 4.19, and $(7)=v(L)$ by Proposition 5.4. 


\section{The restricted numerical dimension}

We now turn to the restricted numerical dimension. For a subvariety $V, v_{X \mid V}(L)$ should measure the maximal dimension of a subvariety $W \subset V$ such that the "positive restriction" of $L$ to $W$ is big.

Theorem 7.1. Let $X$ be a smooth variety, let $V$ be a subvariety of $X$, and let $L$ be a $V$-pseudoeffective divisor. In the following, A denotes some fixed sufficiently ample $\mathbb{Z}$-divisor, and $W$ will range over all subvarieties of $V$ not contained in $\mathbf{B}_{-}(L)$. Then the following quantities coincide:

(1) $\max \left\{k \in \mathbb{Z}_{\geq 0} \mid\left\langle L^{k}\right\rangle_{X \mid V} \neq 0\right\}$,

(2) $\max \left\{\operatorname{dim} W \mid\left\langle L^{\operatorname{dim} W}\right\rangle_{X \mid W}>0\right\}$,

(3) $\max \left\{\operatorname{dim} W \mid \lim _{\epsilon \rightarrow 0} \operatorname{vol}_{X \mid W}(L+\epsilon A)>0\right\}$, and

(4) $\max \left\{\operatorname{dim} W \mid \liminf _{\phi} \operatorname{vol}_{\widetilde{W}}\left(\left.\left[P_{\sigma}\left(\phi^{*} L\right)\right]\right|_{\widetilde{W}}\right)>0\right\}$, where $\phi:(\widetilde{X}, \widetilde{W}) \rightarrow(X, W)$ ranges over $W$-birational models.

This common quantity is known as the restricted numerical dimension of $L$ along $V$ and is denoted $v_{X \mid V}(L)$. It only depends on the numerical class of $L$.

The argument is the same as in the proof of the first four equivalences in Theorem 6.2. One wonders whether the other equalities in Theorem 6.2 can be extended to analogous notions for the restricted numerical dimension. Perhaps the most important is the restricted version of $\kappa_{\sigma}$.

Definition 7.2. Let $X$ be a smooth variety, let $V$ be a subvariety, and let $L$ be a $V$-pseudoeffective divisor. Fix any divisor $A$. If $H^{0}\left(X \mid V, O_{X}(\lfloor m L+A\rfloor)\right)$ is nonzero for infinitely many values of $m$, we define

$$
\kappa_{\sigma}(X \mid V, L ; A):=\max \left\{k \in \mathbb{Z}_{\geq 0} \mid \limsup _{m \rightarrow \infty} \frac{h^{0}\left(X \mid V, \mathrm{O}_{X}(\lfloor m L+A\rfloor)\right)}{m^{k}}>0\right\} .
$$

Otherwise, define $\kappa_{\sigma}(X \mid V, L ; A):=-\infty$. The restricted $\sigma$-dimension $\kappa_{\sigma}(X \mid V, L)$ is defined to be

$$
\kappa_{\sigma}(X \mid V, L):=\max _{A}\left\{\kappa_{\sigma}(X \mid V, L ; A)\right\} .
$$

Arguing as in the proof of [Nakayama 2004, Proposition V.2.7], one can check that the restricted $\sigma$-dimension is a numerical and birational invariant.

Question 7.3. Let $X$ be a smooth variety, $V$ a subvariety, and $L$ a $V$-pseudoeffective divisor. Does $v_{X \mid V}(L)=\kappa_{\sigma}(X \mid V, L)$ ?

Since the restricted numerical dimension is invariant under passing to admissible models, we can extend the definition to pairs with singularities. 
Definition 7.4. Let $X$ be a normal variety, $V$ a subvariety not contained in $\operatorname{Sing}(X)$, and $L$ a $V$-pseudoeffective divisor. We define $v_{X \mid V}(L)=v_{Y \mid W}\left(f^{*} L\right)$, where $(Y, W)$ is any smooth $V$-birational model of $(X, V)$.

7A. Properties of the restricted numerical dimension. The restricted numerical dimension satisfies similar properties to the numerical dimension. Since we know less about $v_{X \mid V}$, the statements are slightly weaker.

Theorem 7.5. Let $X$ be a smooth variety, $V$ a subvariety of $X$, and $L a V$ pseudoeffective divisor.

(1) We have $v_{X \mid V}(L) \leq v(L)$, and if $V$ is normal, then $v_{X \mid V}(L) \leq v\left(\left.L\right|_{V}\right)$.

(2) We have $v_{X \mid V}(L)=v_{X \mid V}\left(P_{\sigma}(L)\right)$.

(3) When $L$ is numerically effective, $v_{X \mid V}(L)=v_{V}\left(\left.L\right|_{V}\right)$.

(4) If $L^{\prime}$ is also $V$-pseudoeffective, then $v_{X \mid V}\left(L+L^{\prime}\right) \geq v_{X \mid V}(L)$.

(5) Suppose that $v_{X \mid V}(L)<\operatorname{dim} V$. If $H$ is a very general, very ample divisor on $X$, then $v_{X \mid V}(L)=v_{X \mid V \cap H}(L)$.

(6) If $\phi:(\widetilde{X}, \widetilde{V}) \rightarrow(X, V)$ is an admissible model with $\widetilde{X}$ smooth, then we have $v_{\tilde{X} \mid \tilde{V}}\left(\phi^{*} L\right)=v_{X \mid V}(L)$.

(7) Let $\phi: Y \rightarrow X$ be a smooth birational model, and let $W$ be a subvariety of $Y$ such that $\left.\phi\right|_{W}$ maps surjectively onto $V$. Then $v_{Y \mid W}\left(\phi^{*} L\right)=v_{X \mid V}(L)$.

Proof. (1) Note that if $Z$ and $Z^{\prime}$ are subvarieties of $X$ with $Z \subset Z^{\prime}$, then we have $\operatorname{vol}_{X \mid Z^{\prime}}(L) \geq \operatorname{vol}_{X \mid Z}(L)$ since the restriction map on sections of $L$ from $X$ to $Z$ factors through the restriction map to $Z^{\prime}$.

Fix an ample divisor $A$ on $X$, and let $W$ be an intersection of very general, very ample divisors on $X$. The two inequalities follow from the two facts that $\operatorname{vol}_{X \mid W}(L+\epsilon A) \geq \operatorname{vol}_{X \mid V \cap W}(L+\epsilon A)$ and $\operatorname{vol}_{X \mid V}(L+\epsilon A) \geq \operatorname{vol}_{X \mid V \cap W}(L+\epsilon A)$.

(2) This follows from the fact that the restricted positive product is invariant under passing to $P_{\sigma}$ as demonstrated in Proposition 4.13.

(3) The restricted volume of an ample divisor can be calculated as an intersection product, so the equality follows from characterization (3) in Theorem 7.1.

(4) Fix an ample divisor $A$. Then the inequality follows from the other inequality $\operatorname{vol}_{X \mid W}\left(L+L^{\prime}+2 \epsilon A\right) \geq \operatorname{vol}_{X \mid W}(L+\epsilon A)$.

(5) Using characterization (1) in Theorem 7.1, we see that if $k<\operatorname{dim} V$, then $\left\langle L^{k}\right\rangle_{X \mid V} \neq 0$ if and only if $\left\langle L^{k}\right\rangle_{X \mid V \cap H}=\left\langle L^{k}\right\rangle_{X \mid V} \cdot H \neq 0$.

(6) This is a consequence of Proposition 4.20 that describes how the restricted positive product is compatible with admissible models.

(7) First suppose that $\operatorname{dim} W>\operatorname{dim} V$; we show $v_{Y \mid W}\left(\phi^{*} L\right)<\operatorname{dim} W$. Every fiber of $\left.\phi\right|_{W}$ is covered by curves with $\phi^{*} L \cdot C=0$. Since $\mathbf{B}_{-}\left(\phi^{*} L\right)=\phi^{-1} \mathbf{B}_{-}(L)$, the 
general such curve avoids $\mathbf{B}_{-}\left(\phi^{*} L\right)$. In particular, for any $W$-birational model $\psi: \widetilde{Y} \rightarrow Y$, the subvariety $\widetilde{W}$ is covered by curves satisfying $P_{\sigma}\left(\psi^{*} \phi^{*} L\right) \cdot C=0$. Thus, $v_{Y \mid W}\left(\phi^{*} L\right)<\operatorname{dim} W$ by characterization (4) in Theorem 7.1.

Fix a very general, very ample divisor $H$ on $Y$. Then $v_{Y \mid W}\left(\phi^{*} L\right)=v_{Y \mid W \cap H}\left(\phi^{*} L\right)$ by property (5). Proceeding inductively, we reduce to the case $\operatorname{dim} W=\operatorname{dim} V$, which is (6).

It is important to note we can have $v_{X \mid V}(L)=\operatorname{dim} V$ even when $L$ is not $V$-big.

Example 7.6. Let $X$ be a smooth variety, $V$ a smooth subvariety, and $L$ a $V$ big divisor. Let $\phi:(Y, W) \rightarrow(X, V)$ be an admissible model such that some $\phi$-exceptional center contains $V$. Then $\phi^{*} L$ is $W$-pseudoeffective but not $W$-big. Nevertheless, the invariance of $v_{X \mid V}(L)$ under passing to admissible models shows that we still have $v_{X \mid V}(L)=\operatorname{dim} V$.

We next show that the nonvanishing of $v(L)$ can be detected by the restricted numerical dimension $v_{X \mid C}(L)$ for a very general curve $C$.

Proposition 7.7. Let $X$ be a smooth variety, and let $L$ be a pseudoeffective divisor on $X$. Then $v(L)>0$ if and only if there is a curve $C$ on $X$ defined as a very general complete intersection of very ample divisors with $v_{X \mid C}(L)>0$.

Proof. If $v(L)=0$, then $v_{X \mid C}(L)=0$ by Theorem 7.5.

Conversely, suppose that $C$ is a very general intersection of very ample divisors. By choosing $C$ appropriately, we may assume that it avoids every component of $\mathbf{B}_{-}\left(P_{\sigma}(L)\right)$. In particular, for any $C$-birational model $\phi: Y \rightarrow X$, we have

$$
\operatorname{vol}\left(\left.P_{\sigma}\left(\phi^{*} L\right)\right|_{\widetilde{C}}\right)=P_{\sigma}\left(\phi^{*} L\right) \cdot \widetilde{C}=\phi^{*} P_{\sigma}(L) \cdot \widetilde{C}=P_{\sigma}(L) \cdot C .
$$

Thus, if $v_{X \mid C}(L)=0$, then $P_{\sigma}(L) \cdot C=0$. But since $C$ is an intersection of ample divisors, this implies that $P_{\sigma}(L) \equiv 0$ and $v(L)=0$.

\section{Acknowledgments}

I would like to thank my advisor J. $\mathrm{M}^{\mathrm{c}}$ Kernan for his advice and support. I also thank R. Lazarsfeld for some helpful conversations, Y. Gongyo for pointing out several mistakes in an earlier draft, and the referee for the careful revisions.

\section{References}

[Boucksom 2004] S. Boucksom, "Divisorial Zariski decompositions on compact complex manifolds", Ann. Sci. École Norm. Sup. (4) 37:1 (2004), 45-76. MR 2005i:32018 Zbl 1054.32010

[Boucksom et al. 2009] S. Boucksom, C. Favre, and M. Jonsson, "Differentiability of volumes of divisors and a problem of Teissier", J. Algebraic Geom. 18:2 (2009), 279-308. MR 2009m:14005 Zbl 1162.14003 
[Boucksom et al. 2012] S. Boucksom, J.-P. Demailly, M. Păun, and T. Peternell, "The pseudo-effective cone of a compact Kähler manifold and varieties of negative Kodaira dimension”, preprint, 2012. To appear in print J. Algebraic Geom.

[Ein et al. 2006] L. Ein, R. Lazarsfeld, M. Mustaţă, M. Nakamaye, and M. Popa, "Asymptotic invariants of base loci”, Ann. Inst. Fourier (Grenoble) 56:6 (2006), 1701-1734. MR 2007m:14008 Zbl 1127.14010

[Ein et al. 2009] L. Ein, R. Lazarsfeld, M. Mustață, M. Nakamaye, and M. Popa, "Restricted volumes and base loci of linear series", Amer. J. Math. 131:3 (2009), 607-651. MR 2010g:14005 Zbl 1179.14006

[Fulton 1984] W. Fulton, Intersection theory, Ergeb. Math. Grenzgeb. (3) 2, Springer, Berlin, 1984. MR 85k:14004 Zbl 0541.14005

[Kawamata 1985] Y. Kawamata, "Pluricanonical systems on minimal algebraic varieties", Invent. Math. 79:3 (1985), 567-588. MR 87h:14005 Zbl 0593.14010

[Lazarsfeld 2004] R. Lazarsfeld, Positivity in algebraic geometry, II: Positivity for vector bundles, and multiplier ideals, Ergeb. Math. Grenzgeb. (3) 49, Springer, Berlin, 2004. MR 2005k:14001b Zbl 1093.14500

[Lazarsfeld and Mustață 2009] R. Lazarsfeld and M. Mustață, "Convex bodies associated to linear series”, Ann. Sci. Éc. Norm. Supér. (4) 42:5 (2009), 783-835. MR 2011e:14012 Zbl 1182.14004

[Nakamaye 2000] M. Nakamaye, "Stable base loci of linear series", Math. Ann. 318:4 (2000), 837-847. MR 2002a:14008 Zbl 1063.14008

[Nakamaye 2003] M. Nakamaye, "Base loci of linear series are numerically determined", Trans. Amer. Math. Soc. 355:2 (2003), 551-566. MR 2003j:14007 Zbl 1017.14017

[Nakayama 2004] N. Nakayama, Zariski-decomposition and abundance, MSJ Memoirs 14, Mathematical Society of Japan, Tokyo, 2004. MR 2005h:14015 Zbl 1061.14018

[Swanson 2000] I. Swanson, "Linear equivalence of ideal topologies", Math. Z. 234:4 (2000), 755775. MR 2001f:13037 Zbl 1010.13015

Communicated by Yujiro Kawamata

Received 2011-09-20 Revised 2012-07-09 Accepted 2012-08-03

blehmann@rice.edu

Department of Mathematics, Rice University,

6100 Main Street, Houston, TX, 77005, United States 


\section{Algebra \& Number Theory}

msp.org/ant

\section{EDITORS}

MANAGING EDITOR

Bjorn Poonen

Massachusetts Institute of Technology

Cambridge, USA

\author{
EDITORIAL BOARD CHAIR \\ David Eisenbud \\ University of California \\ Berkeley, USA
}

\section{BOARD OF EDITORS}

Georgia Benkart

Dave Benson

Richard E. Borcherds

John H. Coates

J-L. Colliot-Thélène

Brian D. Conrad

Hélène Esnault

Hubert Flenner

Edward Frenkel

Andrew Granville

Joseph Gubeladze

Ehud Hrushovski

Craig Huneke

Mikhail Kapranov

Yujiro Kawamata

János Kollár

Yuri Manin

Barry Mazur

Philippe Michel
University of Wisconsin, Madison, USA

University of Aberdeen, Scotland

University of California, Berkeley, USA

University of Cambridge, UK

CNRS, Université Paris-Sud, France

University of Michigan, USA

Freie Universität Berlin, Germany

Ruhr-Universität, Germany

University of California, Berkeley, USA

Université de Montréal, Canada

San Francisco State University, USA

Hebrew University, Israel

University of Virginia, USA

Yale University, USA

University of Tokyo, Japan

Princeton University, USA

Northwestern University, USA

Harvard University, USA

École Polytechnique Fédérale de Lausanne
Susan Montgomery

Shigefumi Mori

Raman Parimala

Jonathan Pila

Victor Reiner

Karl Rubin

Peter Sarnak

Joseph H. Silverman

Michael Singer

Vasudevan Srinivas

J. Toby Stafford

Bernd Sturmfels

Richard Taylor

Ravi Vakil

Michel van den Bergh

Marie-France Vignéras

Kei-Ichi Watanabe

Efim Zelmanov
University of Southern California, USA

RIMS, Kyoto University, Japan

Emory University, USA

University of Oxford, UK

University of Minnesota, USA

University of California, Irvine, USA

Princeton University, USA

Brown University, USA

North Carolina State University, USA

Tata Inst. of Fund. Research, India

University of Michigan, USA

University of California, Berkeley, USA

Harvard University, USA

Stanford University, USA

Hasselt University, Belgium

Université Paris VII, France

Nihon University, Japan

University of California, San Diego, USA

\section{PRODUCTION}

production@msp.org

Silvio Levy, Scientific Editor

See inside back cover or msp.org/ant for submission instructions.

The subscription price for 2013 is US \$200/year for the electronic version, and \$350/year ( $\$ 40$, if shipping outside the US) for print and electronic. Subscriptions, requests for back issues and changes of subscribers address should be sent to MSP.

Algebra \& Number Theory (ISSN 1944-7833 electronic, 1937-0652 printed) at Mathematical Sciences Publishers, 798 Evans Hall \#3840, c/o University of California, Berkeley, CA 94720-3840 is published continuously online. Periodical rate postage paid at Berkeley, CA 94704, and additional mailing offices.

ANT peer review and production are managed by EditFLOW ${ }^{\circledR}$ from Mathematical Sciences Publishers.

\section{PUBLISHED BY}

- mathematical sciences publishers

nonprofit scientific publishing

http://msp.org/

(C) 2013 Mathematical Sciences Publishers 


\section{Algebra \& Number Theory}

Volume $7 \quad$ No. $5 \quad 2013$

Sharp upper bounds for the Betti numbers of a given Hilbert polynomial

GiUlio CAVIGLia and SATOShi Murai

Comparing numerical dimensions

1065

BRIAN LEHMANN

Some consequences of a formula of Mazur and Rubin for arithmetic local constants JAN NEKOVÁŘ

Quantized mixed tensor space and Schur-Weyl duality

RICHARD DIPPER, STEPHEN DOTY and FRIEDERIKE STOLL

Weakly commensurable $S$-arithmetic subgroups in almost simple algebraic groups of

types B and C

SKIP GARIBALDI and ANDREI RAPINCHUK

Minimisation and reduction of 5-coverings of elliptic curves

1179

TOM FISHER

On binary cyclotomic polynomials

1207

ÉTIENNE FOUVRY

Local and global canonical height functions for affine space regular automorphisms

SHU KAWAGUCHI

On the ranks of the 2-Selmer groups of twists of a given elliptic curve

1101

1121

147

DANIEL M. KANE 